IISc-CTS-12/01

FZJ-IKP(Th)-2001-10

IPNO-DR 01-014

November 10, 2018

\title{
$\pi K$ SUM RULES AND THE SU(3) CHIRAL EXPANSION
}

\author{
B. Ananthanarayan \\ Centre for Theoretical Studies \\ Indian Institute of Science \\ Bangalore 560 012, India \\ P. Büttiker \\ Institut für Kernphysik \\ Forschungszentrum Jülich \\ D-52425 Jülich, Germany \\ B. Moussallam \\ Groupe de Physique Théorique, IPN \\ Université Paris-Sud \\ F-91406 Orsay Cédex, France
}

\begin{abstract}
A recently proposed set of sum rules, based on the pion-Kaon scattering amplitudes and their crossing-symmetric conjugates are analysed in detail. A key role is played by the $l=0 \pi \pi \rightarrow K \bar{K}$ amplitude which requires an extrapolation to be performed. It is shown how this is tightly constrained from analyticity, chiral counting and the available experimental data, and its stability is tested. A re-evaluation of the $O\left(p^{4}\right)$ chiral couplings $L_{1}, L_{2}, L_{3}$ is obtained, as well as a new evaluation of the large $N_{c}$ suppressed coupling $L_{4}$.
\end{abstract}




\section{Introduction}

Chiral perturbation theory is a rigorous approach to QCD in a restricted but nonperturbative regime, which has recently been developed to $O\left(p^{6}\right)$, i.e. to the next-to-next to the leading order [1]. Foundations of this method [2] and the abundant work which has followed the basic papers where the NLO theory was defined [3, 国 are summarized in the review [5]. The chiral expansion is based on an effective field theory and, as such, involves an increasing number of coupling constants with increasing chiral order. In $S U(3)$, ten couplings $L_{i}(\mu)$ are involved at $O\left(p^{4}\right)$ and ninety more couplings $C_{i}(\mu)$ appear at the next order. In order to make predictions to $O\left(p^{6}\right)$ accuracy, estimates of the $C_{i}(\mu)$ must be performed but, moreover, the values of the $L_{i}$ may have to be modified, compared to their determination using $O\left(p^{4}\right)$ accuracy. The order of magnitude of such a variation, which reflects the rate of convergence of the expansion in the strange quark mass, can be estimated by comparing several different $O\left(p^{4}\right)$ determinations of the same coupling constants. This is one purpose of the present work in which we propose a new determination of $L_{1}, L_{2}$ and $L_{3}$ from a set of sum rules based on the pion-Kaon amplitude and its expression in ChPT at one-loop [6]. We will compare these results with the previous determination from the $K_{l 4}$ form-factors [7, 8] and the (partial) determination from $\pi \pi$ sum rules [3].

A few $O\left(p^{4}\right)$ coupling constants are still very poorly known, in particular, those which are suppressed in the large $N_{c}$ limit: $L_{4}$ and $L_{6}$. Naively, one may even question whether such a suppression should actually hold, because these couplings were shown to be controlled by physics of the scalar meson resonances [9] which fail to obey simple large $N_{c}$ rules. On a more sophisticated level, one may note that some of the large $N_{c}$ suppressed mechanisms, like internal quark loops, are partly taken into account in the chiral expansion via meson loops. The question remains of what value of the scale $\mu$ is the one at which the suppression operates. Another related interesting issue is that of the phase structure of QCD-like theories as a function of the number $N_{F}$ of massless flavours and the value of $N_{F}^{\text {crit }}$ above which chiral symmetry is no longer spontaneously broken. Some recent lattice simulations [10] have obtained values as small as $N_{F}^{\text {crit }} \simeq 4$. If true, this should affect the $S U(3)$ chiral expansion. For instance, it can be seen that $L_{4}$ and $L_{6}$ control how the chiral order parameters $F_{\pi}$ and $\langle\bar{u} u\rangle$ respectively evolve from $N_{F}=2$ to $N_{F}=3$ [4]. Clearly, a small value of $N_{F}^{c r i t}$, should lead to anomalously large values of $L_{4}, L_{6}$.

In view of this, an interesting outcome of the present work is a determination of $L_{4}$. In principle, it could have been extracted from the $K_{l 4}$ form-factors, but this is not feasible in practice because its contribution is accidentally suppressed [7, 8]. Here, we will take advantage of the fact that no such suppression affects the $\pi K$ amplitude and we will show that an evaluation of $L_{4}$ is then possible, which is at the same level of reliability and accuracy as that of $L_{1}, L_{2}, L_{3}$. Several recent papers have considered aspects of the pion-Kaon scattering amplitudes [11, 12, 13]. One purpose is a better understanding of the scalar resonances (see e.g. 114 for a recent discussion of the experimental situation). This question, of course, is not unrelated to that of the size of the chiral couplings [9].

The dispersive formalism on which the sum rules are based has been developed in a previous paper [15]. This formalism is reviewed in sec.2 below and presented in a 
form suitable for comparison with the $O\left(p^{4}\right)$ expression of the amplitude, which has been computed some time ago by Bernard et al. [6], as well as the $O\left(p^{6}\right)$ expression which should be available in the near future. The detailed form of the sum rules for the $O\left(p^{4}\right)$ coupling constants are then presented in sec.3. The practical evaluation of these sum rules, making use of the available experimental data then presents a difficulty: because of $s-t$ crossing the $\pi \pi \rightarrow K \bar{K}$ amplitude appears and it is needed below the experimentally accessible energy range. This was noted in ref. [15] in which only results not depending on this amplitude were presented. Extrapolation of the $\pi \pi \rightarrow K \bar{K}$ amplitude, in particular for the S-wave, is a problem which was considered a long time ago [16, 17]. We discuss this question in some detail in sec.4, and then present all the results.

\section{Dispersive representation, crossing-symmetry and chiral counting}

Basic work on dispersion relations related to the pion-Kaon amplitudes has been reviewed by Lang [18]. In order to determine the number of subtractions we make the assumption that standard Regge phenomenology applies. As shown in ref. [15] the dispersive representation can be recast in a specific form by taking into account chiral counting. Dropping terms which are of chiral order $O\left(p^{8}\right)$ it can be put in a form which involves functions of only one of the Mandelstam variables $s, t, u$ and are analytic, except for a right-hand cut, plus a polynomial. This was first demonstrated for the case of the pion-pion amplitude in ref. [19]. Let us begin by recalling some basic facts and some notation.

\subsection{Notation and conventions}

Making use of $s-u$ crossing, the two independent isospin $I=1 / 2$ and $I=3 / 2$ pion-Kaon amplitudes can be expressed in terms of the $I=3 / 2$ one,

$$
F^{\frac{1}{2}}(s, t, u)=-\frac{1}{2} F^{\frac{3}{2}}(s, t, u)+\frac{3}{2} F^{\frac{3}{2}}(u, t, s) .
$$

It is convenient to introduce the amplitudes $F^{+}$and $F^{-}$which are respectively even and odd under $s-u$ crossing because they require a different number of subtractions. In terms of isospin amplitudes, they are defined as

$$
\begin{aligned}
& F^{+}(s, t, u)=\frac{1}{3} F^{\frac{1}{2}}(s, t, u)+\frac{2}{3} F^{\frac{3}{2}}(s, t, u) \\
& F^{-}(s, t, u)=\frac{1}{3} F^{\frac{1}{2}}(s, t, u)-\frac{1}{3} F^{\frac{3}{2}}(s, t, u) .
\end{aligned}
$$

Under $s-t$ crossing, one generates the $I=0$ and $I=1 \pi \pi \rightarrow K \bar{K}$ amplitudes,

$$
\begin{aligned}
& G^{0}(t, s, u)=\sqrt{6} F^{+}(s, t, u) \\
& G^{1}(t, s, u)=2 F^{-}(s, t, u) .
\end{aligned}
$$

The partial wave expansion of the $\pi K$ isospin amplitudes are defined as

$$
F^{I}(s, t)=16 \pi \sum_{l}(2 l+1) P_{l}\left(z_{s}\right) f_{l}^{I}(s) .
$$


In a similar way we can expand $F^{+}$and $F^{-}$, the corresponding partial-wave projections are denoted $f_{l}^{+}(s)$ and $f_{l}^{-}(s)$. The s-channel scattering angle appearing above is given by

$$
z_{s}=\frac{s(t-u)+m_{-}^{2} m_{+}^{2}}{\left(s-m_{-}^{2}\right)\left(s-m_{+}^{2}\right)} \quad \text { with } m_{ \pm}=m_{K} \pm m_{\pi} .
$$

The partial-wave expansion of the $\pi \pi \rightarrow K \bar{K}$ amplitude is conventionally defined as,

$$
G^{I}(t, s)=16 \pi \sqrt{2} \sum_{l}(2 l+1) P_{l}\left(z_{t}\right) g_{l}^{I}(t)\left(q_{\pi}(t) q_{K}(t)\right)^{l}
$$

with

$$
q_{P}(t)=\frac{1}{2} \sqrt{t-4 m_{P}^{2}}, \quad z_{t}=\frac{s-u}{4 q_{\pi}(t) q_{K}(t)} .
$$

The rationale for introducing the factor $\left(q_{\pi} q_{K}\right)^{l}$ in eq. (6) is explained by Frazer and Fulco [20]. It ensures that the partial-wave amplitudes $g_{l}^{I}(t)$ have good analytic properties. With these definitions, the partial-wave S-matrices are given by

$$
\begin{aligned}
& \pi K \rightarrow \pi K: S_{l}^{I}(s)=1+2 i \frac{\sqrt{\left(s-m_{-}^{2}\right)\left(s-m_{+}^{2}\right)}}{s} f_{l}^{I}(s) \\
& \pi \pi \rightarrow K \bar{K}: S_{l}^{I}(t)=4 i \frac{\left(q_{\pi}(t) q_{K}(t)\right)^{l+1 / 2}}{\sqrt{t}} g_{l}^{I}(t) .
\end{aligned}
$$

\subsection{Dispersive representation of $F^{+}(s, t)$}

One first writes down a dispersive representation with $t$ fixed (and small). According to Regge phenomenology, the asymptotic dependence as a function of $s$ is controlled by the Pomeron, implying the need for two subtractions, which would also result on the general basis of the Froissart bound,

$$
F^{+}(s, t)=\tilde{c}(t)+\frac{1}{\pi} \int_{m_{+}^{2}}^{\infty} \frac{d s^{\prime}}{\left(s^{\prime}\right)^{2}}\left(\frac{s^{2}}{s^{\prime}-s}+\frac{u^{2}}{s^{\prime}-u}\right) \operatorname{Im} F^{+}\left(s^{\prime}, t\right),
$$

giving $F^{+}(s, t)$ in terms of an unknown function of $t$. Next, following ref. [19], one splits the integration range into two regions a) $\left[m_{+}^{2}, \Lambda^{2}\right]$ and b) $\left[\Lambda^{2}, \infty\right], \Lambda$ being the scale of the chiral expansion, i.e. $\Lambda \simeq 1 \mathrm{GeV}$. In the lower integration range, we can apply the chiral counting and drop the imaginary parts of the partial waves with $l \geq 2$ which are $O\left(p^{8}\right)$, i.e. we put

$$
\operatorname{ImF}^{+}\left(s^{\prime}, t\right)=16 \pi\left[\operatorname{Im} f_{0}^{+}+3 \operatorname{Im} f_{1}^{+}\left(s^{\prime}\right) \frac{s^{\prime}\left(t-u^{\prime}\right)+\Delta_{K \pi}^{2}}{\left(s^{\prime}-m_{-}^{2}\right)\left(s^{\prime}-m_{+}^{2}\right)}\right], s^{\prime}<\Lambda^{2} .
$$

In the region b) we can expand in terms of $s, t, u$ divided by $\Lambda^{2}$ again dropping terms which are $O\left(p^{8}\right)$. After some reshuffling of part a) and absorbing functions of $t$ into $\tilde{c}(t)$, one obtains the fixed $t$ dispersive representation in the form

$$
\begin{aligned}
& F^{+}(s, t)=c(t)+\left[W_{0}^{+}(s)+(t-u) W_{1}^{+}(s)+(s \leftrightarrow u)\right] \\
& -(2 u s) \frac{1}{\pi} \int_{\Lambda^{2}}^{\infty} \frac{d s^{\prime}}{\left(s^{\prime}\right)^{3}}\left(1+3 \frac{\Sigma_{\pi K}-t / 2}{s^{\prime}}\right) \operatorname{ImF}^{+}\left(s^{\prime}, t\right)+O\left(p^{8}\right)
\end{aligned}
$$


In the equations above we have introduced the notation

$$
\Sigma_{P Q}=m_{P}^{2}+m_{Q}^{2}, \quad \Delta_{P Q}=m_{P}^{2}-m_{Q}^{2} .
$$

The functions $W_{0}^{+}(s), W_{1}^{+}(s)$ are analytic except for a right-hand cut and are given in terms of the $S$ and $P$ waves of the pion-Kaon amplitude,

$$
\begin{aligned}
& W_{0}^{+}(s)=16 \int_{m_{+}^{2}}^{\Lambda^{2}} \frac{d s^{\prime}}{s^{\prime}-s}\left(\operatorname{Im} f_{0}^{+}\left(s^{\prime}\right)+\Delta_{K \pi}^{2} \frac{3 I m f_{1}^{+}\left(s^{\prime}\right)}{\left(s^{\prime}-m_{-}^{2}\right)\left(s^{\prime}-m_{+}^{2}\right)}\right) \\
& W_{1}^{+}(s)=16 s \int_{m_{+}^{2}}^{\Lambda^{2}} \frac{d s^{\prime}}{s^{\prime}-s} \frac{3 I m f_{1}^{+}\left(s^{\prime}\right)}{\left(s^{\prime}-m_{-}^{2}\right)\left(s^{\prime}-m_{+}^{2}\right)} .
\end{aligned}
$$

In order to further constrain the function $c(t)$ appearing in eq. (11) we must write down for $F^{+}(s, t)$ a dispersion relation involving the cut in the $t$ variable. A possibility is to use a dispersion relation with $s$ fixed. Alternatively, one can use one with $u s$ fixed, $u s=b$, (hyperbolic dispersion relation) which was shown to have better convergence properties [21]. In this case, the variables $s$ and $u$ are functions of $t$ denoted $s_{b}$ and $u_{b}$,

$$
s_{b}(t)=\Sigma_{\pi K}-\frac{t}{2}+\sqrt{\left(\Sigma_{\pi K}-\frac{t}{2}\right)^{2}-b}, u_{b}(t)=b / s_{b}(t) .
$$

The function $F^{+}\left(s_{b}, t\right)$ is an analytic function of $t$ with a) a right-hand cut $4 m_{\pi}^{2} \leq t<\infty$, and $\mathrm{b})$ a left-hand cut $-\infty<t \leq m_{-}^{2}-b / m_{+}^{2}$. In the following, we will adopt a specific value for $b$,

$$
b \equiv \Delta_{K \pi}^{2}=m_{-}^{2} m_{+}^{2},
$$

which corresponds to backward scattering, $z_{s}=-1$. In that case, the upper limit of the left-hand cut is $t=0$. In the asymptotic regions $t \rightarrow \pm \infty$, it is simple to verify that the dominant divergence is controlled by the $K^{*}$ or $K_{2}^{*}$ Regge trajectories, and it is therefore plausible that a single subtraction is sufficient in this case. The following representation is then obtained 17, 15,

$$
\begin{array}{cl}
F^{+}\left(s_{b}, t\right)=\quad & c_{b}+\frac{1}{\pi} \int_{m_{+}^{2}}^{\infty} \frac{d s^{\prime}}{s^{\prime}}\left(\frac{s_{b}}{s^{\prime}-s_{b}}+\frac{u_{b}}{s^{\prime}-u_{b}}\right) \operatorname{ImF}^{+}\left(s^{\prime}, t_{b}^{\prime}\right) \\
& +\frac{t}{\sqrt{6} \pi} \int_{4 m_{\pi}^{2}}^{\infty} \frac{d t^{\prime} \operatorname{Im} G^{0}\left(t^{\prime}, s_{b}^{\prime}\right)}{t^{\prime}\left(t^{\prime}-t\right)}
\end{array}
$$

where $s_{b}^{\prime} \equiv s_{b}\left(t^{\prime}\right)$ (see eq. (14)) and

$$
t_{b}^{\prime}=2 \Sigma_{\pi K}-s^{\prime}-\frac{b}{s^{\prime}} .
$$

Next, one splits the integration range as before and drops terms which are $O\left(p^{8}\right)$. Equating the representations (11) and (16) then determines the unknown function in the former 
expression leaving just one undetermined constant. Introducing the following notation for the various high-energy integrals which are involved,

$$
\begin{aligned}
& H^{+}(n)=\frac{1}{\pi} \int_{\Lambda^{2}}^{\infty} \frac{d s^{\prime}}{\left(s^{\prime}\right)^{n}} \operatorname{Im}^{+}\left(s^{\prime}, 0\right) \\
& \dot{H}^{+}(n)=\frac{1}{\pi} \int_{\Lambda^{2}}^{\infty} \frac{d s^{\prime}}{\left(s^{\prime}\right)^{n}} \partial_{t} \operatorname{Im} F^{+}\left(s^{\prime}, 0\right) \\
& H_{b}^{+}(n)=\frac{1}{\pi} \int_{\Lambda^{2}}^{\infty} \frac{d s^{\prime}}{\left(s^{\prime}\right)^{n}} \operatorname{Im} F^{+}\left(s^{\prime}, t_{b}^{\prime}\right) \\
& G_{b}^{+}(n)=\frac{1}{\sqrt{6} \pi} \int_{\Lambda^{2}}^{\infty} \frac{d t^{\prime}}{\left(t^{\prime}\right)^{n}} \operatorname{Im} G^{0}\left(t^{\prime}, s_{b}^{\prime}\right),
\end{aligned}
$$

we finally obtain the following dispersive representation for the amplitude $F^{+}(s, t)$ :

$$
\begin{aligned}
& F^{+}(s, t)=C+\left[W_{0}^{+}(s)+(t-u) W_{1}^{+}(s)+(s \leftrightarrow u)\right]+U_{0}(t) \\
& +16 t \int_{m_{+}^{2}}^{\Lambda^{2}} d s^{\prime} \frac{3 I m f_{1}^{+}\left(s^{\prime}\right)}{\left(s^{\prime}-m_{-}^{2}\right)\left(s^{\prime}-m_{+}^{2}\right)}-2 u s\left[H^{+}(3)+t \dot{H}^{+}(3)+3\left(\Sigma_{\pi K}-t / 2\right) H^{+}(4)\right] \\
& +2 b t\left[\dot{H}^{+}(3)-3 / 2 H^{+}(4)\right]+t G_{b}^{+}(2)+t^{2} G_{b}^{+}(3)+t^{3} G_{b}^{+}(4) \\
& -t H_{b}^{+}(2)+t\left(t-4 \Sigma_{\pi K}\right) H_{b}^{+}(3)-t\left(t^{2}-6 t \Sigma_{\pi K}+12 \Sigma_{\pi K}^{2}-3 b\right) H_{b}^{+}(4)+O\left(p^{8}\right) .
\end{aligned}
$$

Apart from a polynomial, this expression involves the functions $W_{0}^{+}(z), W_{1}^{+}(z)$ which are defined in terms of the $S$ and $P$ waves of the $\pi K$ amplitude (see eqs. (13)(23)) and the function $U_{0}(z)$ which is defined in terms of the $S$ wave of the $\pi \pi \rightarrow K \bar{K}$ amplitude,

$$
U_{0}(z)=\frac{16}{\sqrt{3}} z \int_{4 m_{\pi}^{2}}^{\Lambda^{2}} d t^{\prime} \frac{\operatorname{Im} g_{0}^{0}\left(t^{\prime}\right)}{t^{\prime}\left(t^{\prime}-z\right)}
$$

This derivation shows that the specific form of the amplitude eq. (19) must hold in chiral perturbation theory at $O\left(p^{4}\right)$ (which we will check explicitly below) and also at $O\left(p^{6}\right)$.

\subsection{Dispersive representation of $F^{-}(s, t)$}

We proceed in the same way as for $F^{+}(s, t)$ by first writing down a dispersion relation with $t$ fixed, the only difference is that now, the behaviour at large $s$ is dominated by the $K^{*}$ and $K_{2}^{*}$ Regge exchanges and, therefore, a dispersion representation with no subtraction should converge,

$$
F^{-}(s, t)=\frac{1}{\pi} \int_{m_{+}^{2}}^{\infty} d s^{\prime}\left(\frac{1}{s^{\prime}-s}-\frac{1}{s^{\prime}-u}\right) \operatorname{ImF}^{-}\left(s^{\prime}, t\right) .
$$

As before, one splits the integration range into two pieces and in the lower energy range one retains only the $S$ and $P$ waves, obtaining

$$
\begin{aligned}
& F^{-}(s, t)=W_{0}^{-}(s)-W_{0}^{-}(u)+(t-u) W_{1}^{-}(s)-(t-s) W_{1}^{-}(u) \\
& +(s-u)\left[16 \int_{m_{+}^{2}}^{\Lambda^{2}} \frac{3 I m f_{1}^{-}\left(s^{\prime}\right) d s^{\prime}}{\left(s^{\prime}-m_{-}^{2}\right)\left(s^{\prime}-m_{+}^{2}\right)}+\frac{1}{\pi} \int_{\Lambda^{2}}^{\infty} \frac{I m F^{-}\left(s^{\prime}, t\right) d s^{\prime}}{\left(s^{\prime}-s\right)\left(s^{\prime}-u\right)}\right] .
\end{aligned}
$$


The functions $W_{0}^{-}(s)$ and $W_{1}^{-}(s)$ are exactly analogous to their + counterparts defined above,

$$
\begin{aligned}
& W_{0}^{-}(s)=16 \int_{m_{+}^{2}}^{\Lambda^{2}} \frac{d s^{\prime}}{s^{\prime}-s}\left(\operatorname{Im} f_{0}^{-}\left(s^{\prime}\right)+\Delta_{K \pi}^{2} \frac{3 I m f_{1}^{-}\left(s^{\prime}\right)}{\left(s^{\prime}-m_{-}^{2}\right)\left(s^{\prime}-m_{+}^{2}\right)}\right) \\
& W_{1}^{-}(s)=16 s \int_{m_{+}^{2}}^{\Lambda^{2}} \frac{d s^{\prime}}{s^{\prime}-s} \frac{3 I m f_{1}^{-}\left(s^{\prime}\right)}{\left(s^{\prime}-m_{-}^{2}\right)\left(s^{\prime}-m_{+}^{2}\right)} .
\end{aligned}
$$

This representation has no undefined functions but convergence is ensured only for negative values of $t$. One can extend the range of validity in $t$, and also display the cut structure by combining with a hyperbolic dispersion relation. One writes a dispersion relation at fixed $u s=b$ for the function

$$
F^{-}(s, t) /(s-u)
$$

which is even in $s-u$ and thus free of kinematical singularities, and one obtains

$$
\frac{F^{-}\left(s_{b}, t\right)}{s_{b}-u_{b}}=\frac{1}{2 \pi} \int_{4 m_{\pi}^{2}}^{\infty} \frac{d t^{\prime}}{t^{\prime}-t} \operatorname{Im}\left[\frac{G^{1}\left(t^{\prime}, s_{b}^{\prime}\right)}{s_{b}^{\prime}-u_{b}^{\prime}}\right]+\frac{1}{\pi} \int_{m_{+}^{2}}^{\infty} d s^{\prime} \frac{\operatorname{Im} F^{-}\left(s^{\prime}, t_{b}^{\prime}\right)}{\left(s^{\prime}-s_{b}\right)\left(s^{\prime}-u_{b}\right)} .
$$

In the low energy region of the right-hand cut, only the $\mathrm{P}$ wave of the $\pi \pi \rightarrow K \bar{K}$ amplitude will contribute, which generates the function

$$
U_{1}(z)=6 \sqrt{2} \int_{4 m_{\pi}^{2}}^{\Lambda^{2}} d t^{\prime} \frac{I m g_{1}^{1}\left(t^{\prime}\right)}{t^{\prime}-z} .
$$

Equating the fixed $t$ and fixed $u s$ representations gives the following equation, valid for $t \leq 0$

$$
\begin{aligned}
& 32 \int_{m_{+}^{2}}^{\Lambda^{2}} d s^{\prime} \frac{3 I m f_{1}^{-}\left(s^{\prime}\right)}{\left(s^{\prime}-m_{-}^{2}\right)\left(s^{\prime}-m_{+}^{2}\right)}+\frac{1}{\pi} \int_{\Lambda^{2}}^{\infty} d s^{\prime} \frac{\operatorname{Im} F^{-}\left(s^{\prime}, t\right)}{\left(s^{\prime}-s_{b}\right)\left(s^{\prime}-u_{b}\right)}= \\
& U_{1}(t)+\frac{1}{2 \pi} \int_{\Lambda^{2}}^{\infty} d t^{\prime} \frac{\operatorname{Im} G^{1}\left(t^{\prime}, s_{b}^{\prime}\right)}{\left(t^{\prime}-t\right)\left(s_{b}^{\prime}-u_{b}^{\prime}\right)}+\frac{1}{\pi} \int_{\Lambda^{2}}^{\infty} d s^{\prime} \frac{I m F^{-}\left(s^{\prime}, t_{b}^{\prime}\right)}{\left(s^{\prime}-s_{b}\right)\left(s^{\prime}-u_{b}\right)}
\end{aligned}
$$

which relates the $P$-waves in the $\pi K$ and the $\pi \pi \rightarrow K \bar{K}$ channels. Finally, introducing the following notation for the high-energy integrals,

$$
\begin{aligned}
& H^{-}(n)=\frac{1}{\pi} \int_{\Lambda^{2}}^{\infty} \frac{d s^{\prime}}{\left(s^{\prime}\right)^{n}} \operatorname{ImF}^{-}\left(s^{\prime}, 0\right) \\
& H_{b}^{-}(n)=\frac{1}{\pi} \int_{\Lambda^{2}}^{\infty} \frac{d s^{\prime}}{\left(s^{\prime}\right)^{n}} \operatorname{Im} F^{-}\left(s^{\prime}, t_{b}^{\prime}\right) \\
& G_{b}^{-}(n)=\frac{1}{2 \pi} \int_{\Lambda^{2}}^{\infty} \frac{d t^{\prime}}{\left(t^{\prime}\right)^{n-1}\left(s_{b}^{\prime}-u_{b}^{\prime}\right)} \operatorname{Im} G^{1}\left(t^{\prime}, s_{b}^{\prime}\right),
\end{aligned}
$$

we obtain the dispersive representation for $F^{-}(s, t)$, valid up to $O\left(p^{8}\right)$ contributions, in the form,

$$
F^{-}(s, t)=W_{0}^{-}(s)-W_{0}^{-}(u)+(t-u) W_{1}^{-}(s)-(t-s) W_{1}^{-}(u)+(s-u) U_{1}(t)
$$




$$
\begin{aligned}
& +(s-u)\left\{-16 \int_{m_{+}^{2}}^{\Lambda^{2}} d s^{\prime} \frac{3 I m f_{1}^{-}\left(s^{\prime}\right)}{\left(s^{\prime}-m_{-}^{2}\right)\left(s^{\prime}-m_{+}^{2}\right)}+G_{b}^{-}(2)+t G_{b}^{-}(3)+t^{2} G_{b}^{-}(4)\right. \\
& \left.+H_{b}^{-}(2)+\left(2 \Sigma_{\pi K}-t\right) H_{b}^{-}(3)+\left[\left(2 \Sigma_{\pi K}-t\right)^{2}-b\right] H_{b}^{-}(4)+(b-u s) H^{-}(4)\right\} \\
& +O\left(p^{8}\right) .
\end{aligned}
$$

On the right-hand sides of eqs. (19)(29) the dependence on the cutoff $\Lambda$ must cancel: we have verified that it does, up to $O\left(p^{8}\right)$ terms. The dependence upon the parameter $b$ must also cancel. This gives rise to constraints among the $\pi K$ and $\pi \pi \rightarrow K \bar{K}$ amplitudes and their derivatives which we have not explored.

\section{Chiral representation and sum rules}

\subsection{Chiral representation at $O\left(p^{4}\right)$}

First, let us recall, that at the leading chiral order, $O\left(p^{2}\right)$, one has

$$
F^{\frac{1}{2}}(s, t)=\frac{1}{4 f_{\pi}^{2}}\left(4 s+3 t-4 \Sigma_{\pi K}\right), \quad F^{\frac{3}{2}}(s, t)=\frac{1}{4 f_{\pi}^{2}}\left(-2 s+2 \Sigma_{\pi K}\right)
$$

or

$$
F^{+}(s, t)=\frac{1}{4 f_{\pi}^{2}} t, \quad F^{-}(s, t)=\frac{1}{4 f_{\pi}^{2}}(s-u) .
$$

The corresponding $\pi K$ partial waves, are, first for $l=0$

$$
f_{0}^{\frac{1}{2}}(s)=\frac{1}{128 \pi f_{\pi}^{2}}\left(5 s-2 \Sigma_{\pi K}-\frac{3 \Delta_{K \pi}^{2}}{s}\right), \quad f_{0}^{\frac{3}{2}}(s)=\frac{1}{64 \pi f_{\pi}^{2}}\left(-2 s+2 \Sigma_{\pi K}\right),
$$

then for $l=1$

$$
f_{1}^{\frac{1}{2}}(s)=\frac{1}{128 \pi f_{\pi}^{2}}\left(s-2 \Sigma_{\pi K}+\frac{\Delta_{K \pi}^{2}}{s}\right), \quad f_{1}^{\frac{3}{2}}(s)=0
$$

while the partial waves for $l \geq 2$ vanish at this order. In the $\pi \pi \rightarrow K \bar{K}$ channel, the $l=0$ and $l=1$ partial waves are

$$
g_{0}^{0}(t)=\frac{\sqrt{3} t}{64 \pi f_{\pi}^{2}}, \quad g_{1}^{1}(t)=\frac{\sqrt{2}}{48 \pi f_{\pi}^{2}} .
$$

At the NLO order, according to the discussion above, the $\pi K$ amplitudes must have the following form,

$$
\begin{aligned}
& F^{+}(s, t)=\left[\bar{W}_{0}^{+}(s)+(t-u) \bar{W}_{1}^{+}(s)+(s \leftrightarrow u)\right]+\bar{U}_{0}(t) \\
& +\lambda_{1}^{+} t^{2}+\lambda_{2}^{+}(s-u)^{2}+\beta^{+} t+\alpha^{+}
\end{aligned}
$$

and

$$
\begin{aligned}
& F^{-}(s, t)=\left[\bar{W}_{0}^{-}(s)+(t-u) \bar{W}_{1}^{-}(s)-(s \leftrightarrow u)\right]+(s-u) \bar{U}_{1}(t) \\
& +(s-u)\left(\lambda_{1}^{-} t+\beta^{-}\right) .
\end{aligned}
$$


Indeed, the calculation was performed in ChPT at $O\left(p^{4}\right)$ in ref. [6], and it is not difficult to recast their result in the above form. We display the explicit expressions below, which will be used in the derivation of the sum rules. The $W$ functions receive contributions from $\pi K$ and $\eta K$ intermediate states,

$$
\bar{W}_{l}^{ \pm}(s)=\frac{1}{64 f_{\pi}^{4}}\left(W_{l, \pi K}^{ \pm}(s)+W_{l, \eta K}^{ \pm}(s)\right) .
$$

For the $W_{0, P Q}^{+}$functions, one obtains from 6

$$
\begin{aligned}
W_{0, \pi K}^{+}= & {\left[19 s^{2}-28 s \Sigma_{\pi K}+12 \Sigma_{\pi K}^{2}-9 \Delta_{K \pi}^{2}+\frac{2 \Delta_{K \pi}^{2} \Sigma_{\pi K}}{s}+\frac{4 \Delta_{K \pi}^{4}}{s^{2}}\right] \bar{J}_{\pi K}(s) } \\
& -\frac{4 \Delta_{K \pi}^{4}}{s} \bar{J}_{\pi K}^{\prime}(0) \\
W_{0, \eta K}^{+}= & {\left[3 s^{2}-4 s \Sigma_{\pi K}+\frac{4}{3} \Sigma_{\pi K}^{2}+\Delta_{K \pi}^{2}+6 \Delta_{K \pi} \Delta_{K \eta}-\frac{2 \Delta_{K \pi}}{s}\left(\Delta_{K \pi} \Sigma_{K \eta}\right.\right.} \\
& \left.\left.+2 \Delta_{K \eta} \Sigma_{\pi K}\right)+\frac{4 \Delta_{K \pi}^{2} \Delta_{K \eta}^{2}}{s^{2}}\right] \bar{J}_{K \eta}(s)-\frac{4 \Delta_{K \pi}^{2} \Delta_{K \eta}^{2}}{s} \bar{J}_{K \eta}^{\prime}(0) .
\end{aligned}
$$

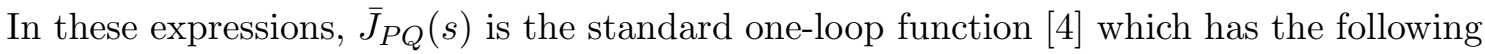
dispersive representation

$$
\bar{J}_{P Q}(s)=\frac{s}{16 \pi^{2}} \int_{\left(m_{P}+m_{Q}\right)^{2}}^{\infty} d s^{\prime} \frac{\sqrt{\lambda_{P Q}\left(s^{\prime}\right)}}{\left(s^{\prime}\right)^{2}\left(s^{\prime}-s\right)},
$$

with

$$
\lambda_{P Q}\left(s^{\prime}\right)=\left(s^{\prime}-\left(m_{P}+m_{Q}\right)^{2}\right)\left(s^{\prime}-\left(m_{P}-m_{Q}\right)^{2}\right) .
$$

Now the $W_{0, P Q}^{-}$functions are

$$
\begin{aligned}
& W_{0, \pi K}^{-}=W_{0, \pi K}^{+}-16\left(s-\Sigma_{\pi K}\right)^{2} \\
& W_{0, \eta K}^{-}=W_{0, \eta K}^{+}
\end{aligned}
$$

The last equality holds because the $\eta K$ state has isospin $I=1 / 2$. Also $I m f_{1}^{\frac{3}{2}}$ vanishes at $O\left(p^{4}\right)$ (and also at $O\left(p^{6}\right)$ ) and consequently,

$$
\bar{W}_{1}^{+}(s)=\bar{W}_{1}^{-}(s) \text {. }
$$

The expression for the $W_{1, P Q}^{+}$components is the same for $P Q=\pi K$ or $\eta K$ and is given by

$$
W_{1, P Q}^{+}=\left(s-2 \Sigma_{P Q}+\frac{\Delta_{P Q}^{2}}{s}\right) \bar{J}_{P Q}(s)-4 \Delta_{P Q}^{2} \bar{J}_{P Q}^{\prime}(0) \text {. }
$$

Finally, the $\bar{U}_{l}$ functions at $O\left(p^{4}\right)$ are

$$
\begin{aligned}
& 16 f_{\pi}^{4} \bar{U}_{0}(t)=2 t\left(2 t-m_{\pi}^{2}\right) \bar{J}_{\pi \pi}(t)+3 t^{2} \bar{J}_{K K}(t)+2 m_{\pi}^{2}\left(t-\frac{8}{9} m_{K}^{2}\right) \bar{J}_{\eta \eta}(t), \\
& 48 f_{\pi}^{4} \bar{U}_{1}(t)=2\left(t-4 m_{\pi}^{2}\right) \bar{J}_{\pi \pi}(t)+\left(t-4 m_{K}^{2}\right) \bar{J}_{K K}(t) .
\end{aligned}
$$


The imaginary parts of the $\bar{W}$ and $\bar{U}$ functions at $O\left(p^{4}\right)$ can be recovered from the definitions eqs. (13) (23) in terms of the imaginary parts of $f_{l}^{ \pm}(s), g_{0}^{0}(s)$ and $g_{1}^{1}(s)$ and using unitarity to relate the latter to the $l=0,1$ amplitudes $\pi K \rightarrow \pi K, \eta K$ and $\pi \pi \rightarrow \pi \pi, K \bar{K}, \eta \eta$ computed at $O\left(p^{2}\right)$. For instance, unitarity gives

$$
\frac{1}{64 f_{\pi}^{4}} \operatorname{Im} W_{0, \pi K}^{+}(s)=16 \pi \frac{\sqrt{\lambda_{\pi K}(s)}}{s}\left[\frac{1}{3}\left|f_{0}^{\frac{1}{2}}(s)\right|^{2}+\frac{2}{3}\left|f_{0}^{\frac{3}{2}}(s)\right|^{2}+\frac{\Delta_{K \pi}}{\lambda_{\pi K}(s)}\left|f_{1}^{\frac{1}{2}}(s)\right|^{2}\right],
$$

and using the $O\left(p^{2}\right)$ expressions (32) (33) for the partial waves one recovers the same imaginary part as in eq. (38).

The separation in eqs. (35) (36) into a polynomial part and a part with cut-analytic functions is arbitrary: we have only required that each piece be scale independent (a different choice was made in ref. [15]) and finite. The coefficients of the polynomials are simple linear functions of the coupling-constants $L_{i}(\mu)$. Using the following notation,

$$
L_{P}=\log \frac{m_{P}^{2}}{\mu^{2}}, R_{P Q}=\frac{m_{P}^{2}}{m_{P}^{2}-m_{Q}^{2}} \log \frac{m_{P}^{2}}{m_{Q}^{2}}
$$

and the result of ref. [6] one finds for the coefficients entering the $F^{-}$amplitude at $O\left(p^{4}\right)$

$$
\begin{aligned}
& f_{\pi}^{2} \beta^{-}=\frac{1}{4}+\frac{2 m_{\pi}^{2}}{f_{\pi}^{2}}\left[L_{5}-\frac{1}{512 \pi^{2}}\left(6 L_{K}+5 R_{\pi K}+R_{\eta K}\right)\right] \\
& f_{\pi}^{4} \lambda_{1}^{-}=-L_{3}+\frac{1}{512 \pi^{2}}\left[-\frac{4}{3} \log \frac{m_{\pi}^{2}}{m_{K}^{2}}+R_{\eta K}+R_{\pi K}\right] .
\end{aligned}
$$

The coefficients entering the $F^{+}$amplitude, then, have the following expression in terms of the $L_{i}$ 's,

$$
\begin{aligned}
& \alpha^{+}=\frac{8 m_{\pi}^{2} m_{K}^{2}}{f_{\pi}^{4}}\left\{4 L_{1}+L_{3}-4 L_{4}-L_{5}+4 L_{6}+2 L_{8}\right. \\
& \left.\quad+\frac{1}{512 \pi^{2}}\left[\frac{7}{9} L_{\eta}-L_{K}-R_{\pi K}+\frac{1}{3} R_{K \eta}-\frac{2}{9}\right]\right\} \\
& \beta^{+}=\beta^{-}+\frac{8\left(m_{\pi}^{2}+m_{K}^{2}\right)}{f_{\pi}^{4}}\left[-2 L_{1}-\frac{1}{2} L_{3}+L_{4}+\frac{1}{512 \pi^{2}}\left(L_{K}+R_{\pi K}+\frac{1}{3}\right)\right] \\
& +\frac{m_{\pi}^{2}}{128 \pi^{2} f_{\pi}^{4}} \log \frac{m_{\pi}^{2}}{m_{\eta}^{2}} \\
& f_{\pi}^{4} \lambda_{1}^{+}=8 L_{1}+2 L_{2}+\frac{5}{2} L_{3}+\frac{1}{512 \pi^{2}}\left[-8 L_{\pi}-10 L_{K}-4 R_{\pi K}-15\right] \\
& f_{\pi}^{4} \lambda_{2}^{+}=2 L_{2}+\frac{1}{2} L_{3}+\frac{1}{512 \pi^{2}}\left[-6 L_{K}-5 R_{\pi K}-R_{\eta K}+\frac{1}{3}\right] .
\end{aligned}
$$

This completes the rewriting of the chiral formulas of ref. [6] in a form which allows easy matching with the dispersive representations. This matching generates a number of sum rules. For $F^{-}$, the dispersive formula has no subtraction constant, which implies that the two coefficients $\beta^{-}$and $\lambda_{1}^{-}$can be expressed as sum rules. For $F^{+}$, one subtraction 
constant remains and this implies that the three coefficients $\beta^{+}, \lambda_{1}^{+}, \lambda_{2}^{+}$are expressible as sum rules while the fourth one, $\alpha^{+}$, remains undetermined in this approach. Using eqs. (47) (48) it is then easy to generate sum rule expressions for the $L_{i}$ coupling constants, which are given in terms of $\beta^{ \pm}, \lambda_{i}^{ \pm}$as simple linear combinations. For instance, $L_{1}, L_{2}$ are given by

$$
\begin{aligned}
& L_{1}=\frac{f_{\pi}^{4}}{8}\left(\lambda_{1}^{+}-\lambda_{2}^{+}+2 \lambda_{1}^{-}\right)-\frac{1}{512 \pi^{2}}\left(-\frac{4}{3} L_{\pi}-\frac{1}{6} L_{K}+\frac{3}{8} R_{\pi K}+\frac{3}{8} R_{\eta K}-\frac{23}{12}\right) \\
& L_{2}=\frac{f_{\pi}^{4}}{4}\left(2 \lambda_{2}^{+}+\lambda_{1}^{-}\right)-\frac{1}{512 \pi^{2}}\left(-\frac{1}{3} L_{\pi}-\frac{8}{3} L_{K}-\frac{9}{4} R_{\pi K}-\frac{1}{4} R_{\eta K}+\frac{1}{6}\right) .
\end{aligned}
$$

while $L_{3}$ is immediately given in terms of $\lambda_{1}^{-}$. The coupling $L_{4}$, finally, is obtained from the following combination

$$
\begin{aligned}
L_{4} & =\frac{f_{\pi}^{4}}{8}\left(\frac{\beta^{+}-\beta^{-}}{m_{K}^{2}+m_{\pi}^{2}}+2\left(\lambda_{1}^{+}-\lambda_{2}^{+}\right)\right) \\
& -\frac{1}{512 \pi^{2}}\left(-2 L_{\pi}+\frac{5}{4} R_{\pi K}+\frac{1}{4} R_{\eta K}-\frac{7}{2}+\frac{m_{\pi}^{2}}{2\left(m_{\pi}^{2}+m_{K}^{2}\right)} \log \frac{m_{\pi}^{2}}{m_{\eta}^{2}}\right) .
\end{aligned}
$$

We observe that while the coupling $L_{5}$ is present in the expression for $\beta^{-}$, it appears multiplied by $m_{\pi}^{2}$ (not $m_{K}^{2}$ ) and thus makes a very small correction to the leading $O\left(p^{2}\right)$ contribution.

\subsection{Sum rules}

The dispersive representation of the $\pi K$ amplitudes eqs. (19) (29) contains one arbitrary parameter, while the polynomial part of the chiral representation eqs. (35) (36) at $O\left(p^{4}\right)$, contains six coefficients: comparing the two representations should yield five sum rules for these coefficients which will translate, in principle, using expressions (48) (47) into sum rules for the five coupling constants $L_{i}(\mu), i=1,5$. The explicit form of the sum rules are obtained by noting that differences like $W_{0}^{+}(s)-\bar{W}_{0}^{+}(s)$, in which $\bar{W}_{0}^{+}$is computed to $O\left(p^{4}\right)$ accuracy, are analytic up to $O\left(p^{6}\right)$ contributions,

$$
\operatorname{Im}\left(W_{0}^{+}(s)-\bar{W}_{0}^{+}(s)\right)=O\left(p^{6}\right) .
$$

Therefore, up to $O\left(p^{6}\right)$ terms, we can expand these differences as polynomials,

$$
\begin{aligned}
& W_{l}^{ \pm}(z)-\bar{W}_{l}^{ \pm}(z)=A_{l}^{ \pm}+B_{l}^{ \pm} z+C_{l}^{ \pm} z^{2} \\
& U_{l}(z)-\bar{U}_{l}(z)=u_{l}+v_{l} z+w_{l} z^{2}
\end{aligned}
$$

for $l=0,1$. We also introduce

$$
\hat{A}_{1}^{ \pm}=16 \int_{m_{+}^{2}}^{\Lambda^{2}} \frac{3 I m f_{1}^{ \pm}\left(s^{\prime}\right) d s^{\prime}}{\left(s^{\prime}-m_{+}^{2}\right)\left(s^{\prime}-m_{-}^{2}\right)} .
$$

The coefficients $A_{l}^{ \pm}, B_{l}^{ \pm}$etc... are given as inverse moments of the imaginary parts of the $\pi K$ and $\pi \pi \rightarrow K \bar{K} S$ and $P$ waves, integrated between the threshold and $\Lambda^{2}$, with the 
chiral part being subtracted. Together with the integrals $(18)(\sqrt{28})$ over the high-energy region, $\left[\Lambda^{2}, \infty\right]$, they form the building blocks of the sum rules.

Equating the chiral and the dispersive expressions, taking into account eqs. (52), we finally obtain the following sum rule formulas for the polynomial coefficients in the $O\left(p^{4}\right)$ chiral representation (35) (36)

$$
\begin{aligned}
\beta^{-}= & -\hat{A}_{1}^{-}+A_{1}^{-}+B_{0}^{-}+u_{1}+2 \Sigma_{\pi K} C_{0}^{-}+G_{b}^{-}(2)+H_{b}^{-}(2)+2 \Sigma_{\pi K} H_{b}^{-}(3) \\
\beta^{+}= & \hat{A}_{1}^{+}+3 A_{1}^{+}-B_{0}^{+}+v_{0}+2 \Sigma_{\pi K}\left(2 B_{1}^{+}-C_{0}^{+}\right)+G_{b}^{+}(2) \\
& -H_{b}^{+}(2)+2 \Sigma_{\pi K}\left(H^{+}(3)-2 H_{b}^{+}(3)\right) \\
\lambda_{1}^{+}= & -\frac{3}{2} B_{1}^{+}+\frac{1}{2} C_{0}^{+}+w_{0}+G_{b}^{+}(3)-\frac{1}{2} H^{+}(3)+H_{b}^{+}(3) \\
\lambda_{2}^{+}= & \frac{1}{2} B_{1}^{+}+\frac{1}{2} C_{0}^{+}+\frac{1}{2} H^{+}(3) \\
\lambda_{1}^{-}= & B_{1}^{-}-C_{0}^{-}+v_{1}+G_{b}^{-}(3)-H_{b}^{-}(3)
\end{aligned}
$$

The derivation and the structure of these sum rules are very similar to those which were proposed for $\pi \pi$ scattering in ref. [22]. We now discuss the practical evaluation of these formulas.

\section{Evaluation of the sum rules}

\section{$4.1 \pi K$ amplitudes}

We will make use of the two most recent high-statistics $K p$ production experiments, both performed at SLAC, which have determined $\pi K$ amplitudes. Estabrooks et al. [23] have considered several charge combinations enabling them to determine separately the $I=1 / 2$ and the $I=3 / 2$ combinations. For the isospin $I=3 / 2$ it was observed that the $\mathrm{P}$ and $\mathrm{D}$ waves remain very small below $\sqrt{s}=2 \mathrm{GeV}$ : in our calculations we will only include the S-wave. A few years later the $K^{-} \pi^{+} \rightarrow K^{-} \pi^{+}$amplitude was remeasured in a slightly larger energy range by Aston et al. [24]. For the $I=1 / 2 \mathrm{~S}$ and $\mathrm{P}$ waves, we have performed fits of the data of Aston et al. with parametrisations in terms of Breit-Wigner plus background similar to those used in this reference using the $I=3 / 2 \mathrm{~S}$-wave from ref. [23]. In these fits, we have imposed that the scattering lengths be equal to their values in ChPT [6]. Relaxing this constraint, however, makes very little change in the results. For the partial waves $l=2-5$, we have used exactly the same parametrisations as provided in ref. 24]. Above $\sqrt{s}=1.5 \mathrm{GeV}$, ambiguities arise in the determination of the $S$ and $P$ waves. Estabrooks et al find four different solutions and Aston et al. two. It has been pointed out in ref. [13] that one of these violate the unitarity bound; therefore, we have used the remaining one. In our sum rules, we note that the contribution from the $S$ and $P$ waves in this energy region becomes negligibly small anyway. Above the energy range covered by these experiments we use Regge parametrisations which we will discuss in more detail below.

\section{$4.2 \pi \pi \rightarrow K \bar{K}$ amplitudes}


Let us first discuss the S-wave amplitude,

$$
g_{0}^{0}(t) \equiv\left|g_{0}^{0}(t)\right| \exp \left(i \phi_{S}(t)\right)
$$

which is a crucial ingredient in the sum rules and is needed for $t \geq 4 m_{\pi}^{2}$ while it is measured only in the range $t \geq 4 m_{K}^{2}$. Analyticity, as is well known [16, 17, 25, 26], is the key to performing this extrapolation. To start with, the phase of the amplitude, $\phi_{S}(t)$, may be considered as known in the whole energy region of interest. Firstly, in the region where $\pi \pi$ scattering is elastic, $\phi_{S}$ is identical to the $\pi \pi$ phase shift (modulo $\pi$ ) from Watson's theorem. It is now well established that, to a very good approximation, the domain where $\pi \pi$ scattering is effectively elastic extends up to the $K \bar{K}$ threshold (see e.g. [27]). Above this point, $\phi_{S}(t)$ has been measured in experiments, we will use the two most recent ones: Cohen et al. [28] (who considered $K^{+} K^{-}$production) and Etkin et al. [29, 30] (who considered $\left.K_{S}^{0} K_{S}^{0}\right)$. These data are shown in Fig. 1 together with the curves which will be used in the calculations. One observes that the two data sets are compatible except very

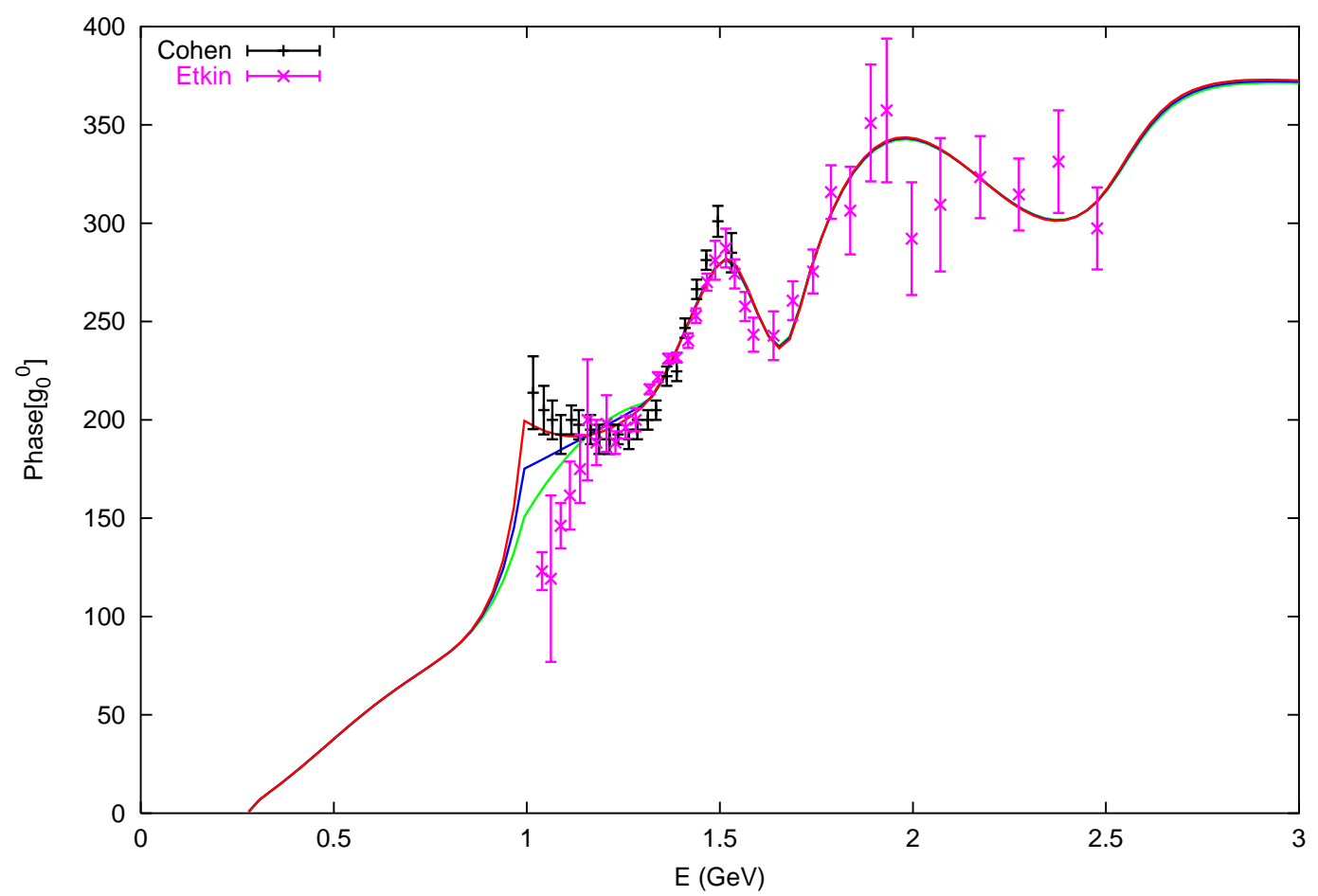

Figure 1: Phase of the $\pi \pi \rightarrow K \bar{K} l=0$ amplitude. The curves are as described in the text. The three curves from bottom to top correspond to three increasing input values for the phase at the $K \bar{K}$ threshold, $\phi_{S}\left(t_{0}\right)=150^{\circ}, 175^{\circ}, 200^{\circ}$.

close to the $K \bar{K}$ threshold. (It must be recalled here that experiments actually measure $S-D$ interference and thus determine only the difference $\phi_{S}-\phi_{D}$. In treating the data of Etkin et al. we have used the $D$-wave model of ref. [31] rather than that used in the 
original paper 29].) In the energy region $\sqrt{t}>2 m_{K}$, we fit the combined set of data with piecewise polynomials. In performing the fit, we have excluded the small energy region where the two data sets are incompatible and we have instead fixed the value of the phase at threshold $\phi_{S}\left(4 m_{K}^{2}\right)$, which we have allowed to vary between $150^{\circ}$ and $220^{\circ}$. This may seem like a wide range and one could think of making use of the equality between $\phi_{S}$ and the $\pi \pi$ phase-shift at the threshold to improve on that. However, the available $\pi \pi$ data points closest to the $K \bar{K}$ threshold have large error bars. In order to get exactly at the threshold, one needs to perform a fit and the result is uncertain because the $\pi \pi$ phase-shift varies extremely rapidly in this region [27]. One ends up with the same range of values as we have chosen. In the energy range $\sqrt{t} \leq 0.8 \mathrm{GeV}$, the curve in Fig. 1 is the result from the recent Roy equations analysis of ref. [32], with $a_{0}^{0}=0.22, a_{0}^{2}=-0.0444$. Because of the new $K_{l 4}$ data [33] there is now a rather small uncertainty on the curve in this energy region [34]. In the energy region between $0.8 \mathrm{GeV}$ and the $K \bar{K}$ threshold we use the simple interpolation formula,

$$
\phi_{S}(t)=\alpha+\frac{\beta}{\sqrt{t}-E_{1}}
$$

in which the three parameters are determined from imposing continuity at both ends and continuity of the first derivative at the lower end.

Once the phase is known, determining the modulus in the region $\left[4 m_{\pi}^{2}, 4 m_{K}^{2}\right]$ is a standard Muskhelishvili-Omnès [35, 36] problem because $g_{0}^{0}$ satisfies the following integral equation

$$
g_{0}^{0}(t)=\frac{t}{\pi} \int_{4 m_{\pi}^{2}}^{\infty} \frac{\operatorname{Im} g_{0}^{0}\left(t^{\prime}\right) d t^{\prime}}{t^{\prime}\left(t^{\prime}-t\right)}+g_{0}^{0}(0)+\Delta(t)
$$

where $\Delta(t)$ has only a left-hand cut. $\Delta(t)$ can be expressed explicitly in terms of $\pi K$ partial wave amplitudes and $\pi \pi \rightarrow K \bar{K}$ partial waves with $l \geq 2$,

$$
\Delta(t)=\sum_{l} \int_{m_{+}^{2}}^{\infty} d s^{\prime} K_{l 0}\left(s^{\prime}, t\right) I m f_{l}^{+}\left(s^{\prime}\right)+\sum_{l \geq 2, \text { even }} \int_{4 m_{\pi}^{2}}^{\infty} d t^{\prime} G_{l 0}\left(t^{\prime}, t\right)\left(q_{\pi} q_{K}\right)^{l} I m g_{l}^{0}\left(t^{\prime}\right) .
$$

For $t \lesssim 1 \mathrm{GeV}^{2}, \Delta(t)$ is dominated by the $\pi K S$ and $P$ waves and eq. (57) is one component of a system of Roy-type equations [37, 38]. This system was expressed in ref. [15] in terms of the two scattering lengths $a_{0}^{1 / 2}, a_{0}^{3 / 2}$. As we do not attempt to solve the full system here, we find it more convenient to use $g_{0}^{0}(0)$ as subtraction constant. We have included $D$ waves as well into the calculation in order to extend the validity of the evaluation of $\Delta(t)$ somewhat above one $\mathrm{GeV}$. The kernels needed in eq. (58) are

$$
\begin{aligned}
& K_{00}\left(s^{\prime}, t\right)=I_{0}\left(s^{\prime}, t\right)-I_{0}\left(s^{\prime}, 0\right), \\
& \text { with } I_{0}\left(s^{\prime}, t\right)=\frac{4}{\sqrt{\left(t-4 m_{\pi}^{2}\right)\left(t-4 m_{K}^{2}\right)}} \operatorname{Arcth} \frac{\sqrt{\left(t-4 m_{\pi}^{2}\right)\left(t-4 m_{K}^{2}\right)}}{2 s^{\prime}-2 \Sigma_{\pi K}+t} \\
& K_{10}\left(s^{\prime}, t\right)=3\left[I_{0}\left(s^{\prime}, t\right)\left(1+\frac{2 s^{\prime} t}{\lambda_{\pi K}\left(s^{\prime}\right)}\right)-\frac{2 t}{\lambda_{\pi K}\left(s^{\prime}\right)}-I_{0}\left(s^{\prime}, 0\right)\right]
\end{aligned}
$$




$$
\begin{aligned}
& K_{20}\left(s^{\prime}, t\right)=5\left[I_{0}\left(s^{\prime}, t\right)\left(1+\frac{6 s^{\prime} t}{\lambda_{\pi K}\left(s^{\prime}\right)}+\frac{6\left(s^{\prime} t\right)^{2}}{\lambda_{\pi K}^{2}\left(s^{\prime}\right)}\right)\right. \\
& \left.+\frac{6 t}{\lambda_{\pi K}^{2}\left(s^{\prime}\right)}\left(s^{\prime}\left(-2 s^{\prime}+2 \Sigma_{\pi K}-t\right)+\frac{1}{6}\left(t-4 m_{\pi}^{2}\right)\left(t-4 m_{K}^{2}\right)\right)-I_{0}\left(s^{\prime}, 0\right)\right] \\
& G_{20}\left(t^{\prime}, t\right)=\frac{16 \times 5}{\sqrt{3}} \frac{t^{\prime}\left(t^{\prime}+t-4 \Sigma_{\pi K}\right)}{t^{\prime}\left(t^{\prime}-4 m_{\pi}^{2}\right)\left(t^{\prime}-4 m_{K}^{2}\right)} .
\end{aligned}
$$

The various contributions and the result for $\Delta(t)$ are displayed in Fig. 2.

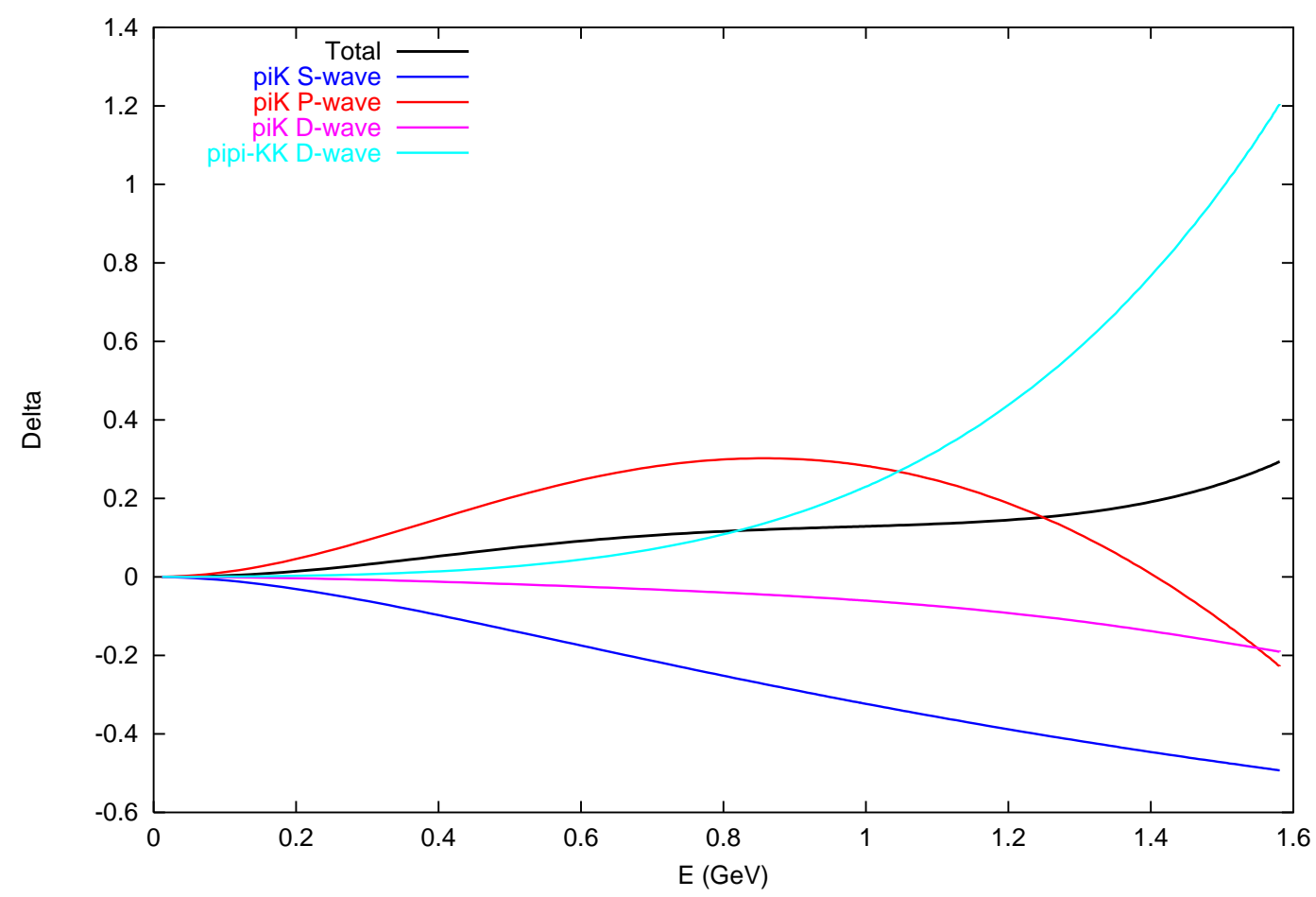

Figure 2: Left-hand cut function, $\Delta(t)$, and its various contributions.

In order to solve equation (57) we first construct the Omnès function over the range $\left[4 m_{\pi}^{2}, t_{0}\right]$, with $t_{0}=4 m_{K}^{2}$

$$
\Omega(t)=\exp \left[\frac{t}{\pi} \int_{4 m_{\pi}^{2}}^{t_{0}} \frac{\phi_{S}\left(t^{\prime}\right) d t^{\prime}}{t^{\prime}\left(t^{\prime}-t\right)}\right] \equiv \Omega_{R}(t) \exp \left[i \phi_{S}(t) \theta\left(t-4 m_{\pi}^{2}\right) \theta\left(t_{0}-t\right)\right]
$$

where $\Omega_{R}(t)$ is real. When $t$ approaches the $K \bar{K}$ threshold, $\Omega_{R}(t)$ has the following behaviour,

$$
\lim _{t \rightarrow 4 m_{K}^{2}} \Omega_{R}(t) \sim\left|t-4 m_{K}^{2}\right|^{\frac{\phi_{S}\left(4 m_{K}^{2}\right)}{\pi}} .
$$


Therefore, two cases must be considered depending whether $\phi_{S}\left(4 m_{K}^{2}\right)$ is smaller or larger than $\pi$. Let us first consider the case

$$
\phi_{S}\left(4 m_{K}^{2}\right) \leq \pi
$$

The solution to eq. (57) is obtained by introducing the function

$$
f(t)=\frac{1}{\Omega(t)}\left(g_{0}^{0}(t)-\Delta(t)\right)
$$

and noting that it is analytic except for a right-hand cut. It can thus be expressed as a dispersion relation which is defined up to a polynomial which depends on the behaviour at infinity of $f(t)$ [35]. We will assume that $f(t)$ is bounded at infinity by a polynomial of degree one and thus, $g_{0}^{0}$ can be expressed in terms of two subtraction constants,

$$
\begin{aligned}
& g_{0}^{0}(t)=\Delta(t)+\Omega(t)\left[\alpha_{0}+\beta_{0} t+\frac{t^{2}}{\pi} \int_{4 m_{\pi}^{2}}^{4 m_{K}^{2}} d t^{\prime} \frac{\Delta\left(t^{\prime}\right) \sin \phi_{S}\left(t^{\prime}\right)}{\Omega_{R}\left(t^{\prime}\right)\left(t^{\prime}\right)^{2}\left(t^{\prime}-t\right)}\right. \\
&\left.+\frac{t^{2}}{\pi} \int_{4 m_{K}^{2}}^{\infty} d t^{\prime} \frac{\left|g_{0}^{0}\left(t^{\prime}\right)\right| \sin \phi_{S}\left(t^{\prime}\right)}{\Omega_{R}\left(t^{\prime}\right)\left(t^{\prime}\right)^{2}\left(t^{\prime}-t\right)}\right] .
\end{aligned}
$$

One observes that the integrals converge at $t^{\prime}=4 m_{K}^{2}$ if the condition (62) is satisfied. A small calculation shows that at $t=4 m_{K}^{2}$ the condition $\left(g_{0}^{0}\right)_{\text {output }}=\left(g_{0}^{0}\right)_{\text {input }}$ is automatically satisfied in eq. (64). Concerning the parameters $\alpha_{0}, \beta_{0}$ it is not difficult to see that for the purpose of using $g_{0}^{0}$ with $O\left(p^{4}\right)$ precision it is consistent to use the values of $\alpha_{0}, \beta_{0}$ with $O\left(p^{2}\right)$ precision, i.e.

$$
\alpha_{0}=0 \quad \beta_{0}=\frac{\sqrt{3}}{64 \pi f_{\pi}^{2}}-\Delta^{\prime}(0)
$$

and $g_{0}^{0}$ gets fully determined (the value of the derivative $\Delta^{\prime}(0)$ is determined numerically to be $\left.\Delta^{\prime}(0) \simeq 0.256 \mathrm{GeV}^{-2}\right)$. The influence of $\Delta(t)$ is illustrated in Fig.3 which compares the full solution from eq. (64) to the solution with $\Delta$ set equal to zero. One sees that in the energy region where we really need to use eq. (64), i.e. below the $K \bar{K}$ threshold $\Delta(t)$ actually has a rather small effect. The solution is essentially controlled from the chiral constraints at $t=0$ and the experimental input at $t \geq 4 m_{K}^{2}$. Above the $K \bar{K}$ threshold, the agreement of the experimental data with the output from eq. (64) seems much improved if $\Delta(t)$ is included. In the case where

$$
\phi_{S}\left(4 m_{K}^{2}\right)>\pi,
$$

we need only modify the definition of $f(t)$ (eq. (63)) to

$$
f(t)=\frac{t-4 m_{K}^{2}}{\Omega(t)}\left(g_{0}^{0}(t)-\Delta(t)\right)
$$

and make the corresponding change in the preceeding formulas. Because of the extra factor of $t$ one needs to introduce one more subtraction, and an additional parameter, $\gamma_{0}$ appears in the solution: we simply fix $\gamma_{0}$ so that input-output agreement is retained in the physical region when the condition (66) holds. 


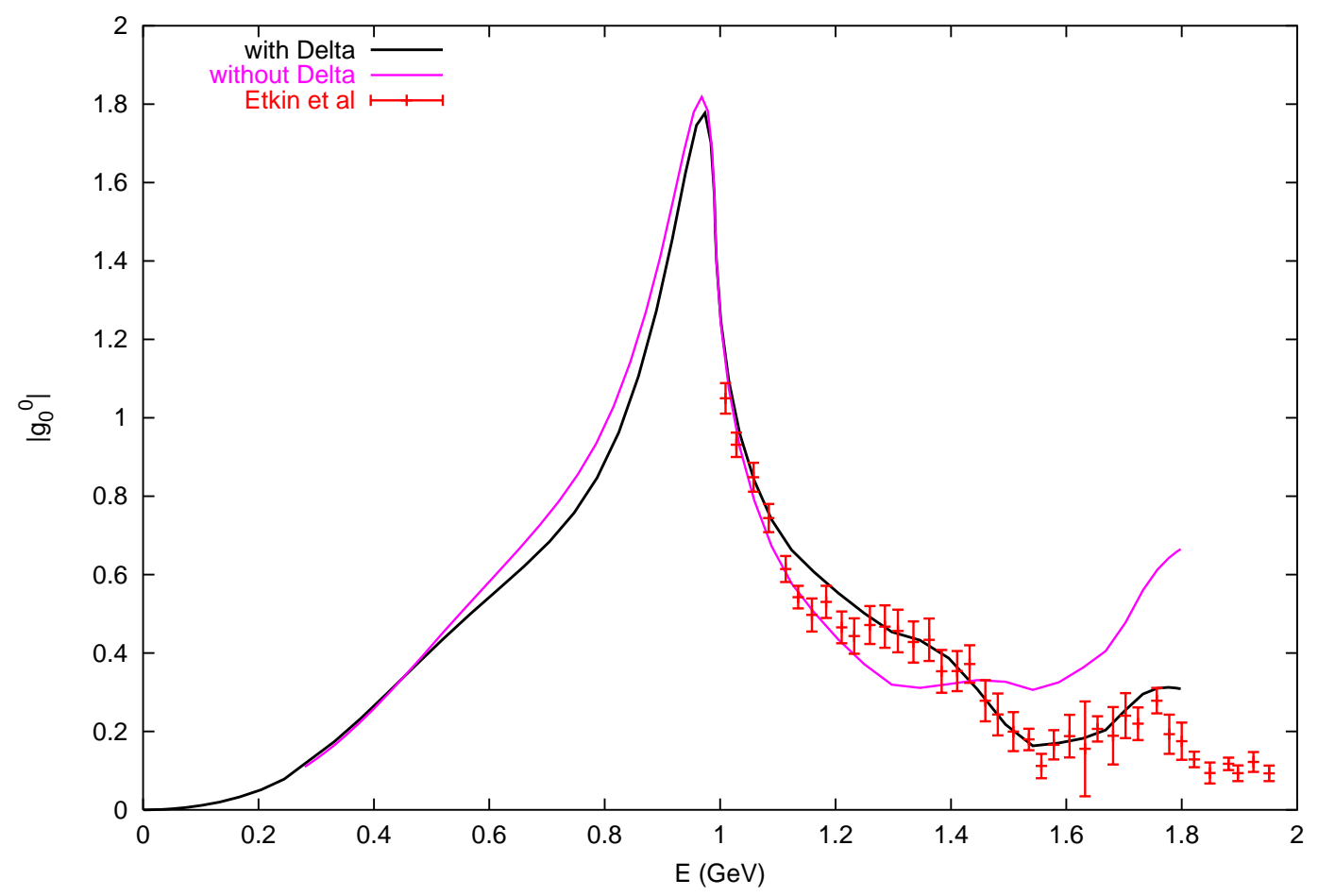

Figure 3: Solutions of eq. (57) with $\Delta(t)$ included and $\Delta(t)$ ignored.

There is a subtlety in the above calculation which must be discussed. Clearly, because of the singular behaviour of the term $1 / \Omega_{R}\left(t^{\prime}\right)$ in the integrand at the $K \bar{K}$ threshold the result will be rather sensitive to the value of $\left|g_{0}^{0}\left(t^{\prime}\right)\right|$ in this region and, in particular, to its value exactly at the threshold, while experimental information starts slightly above the $K \bar{K}$ threshold. A simple solution is to use $t_{0}$ slightly larger than $4 m_{K}^{2}$ in eq. (60). We have done so and found good stability of the result. We have also used the following simple method which, at the same time, provides an alternative extrapolation method in the whole region of interest. We first construct an Omnès function over a region $\left[4 m_{\pi}^{2}, t_{1}\right]$ with $t_{1}>>4 m_{K}^{2}$,

$$
\Omega_{1}(t)=\exp \left[\frac{t}{\pi} \int_{4 m_{\pi}^{2}}^{t_{1}} \frac{\phi_{S}\left(t^{\prime}\right) d t^{\prime}}{t^{\prime}\left(t^{\prime}-t\right)}\right],
$$

and then consider the function

$$
V_{0}^{0}(t)=\frac{1}{\Omega_{1}(t)} g_{0}^{0}(t)
$$

The function $V_{0}^{0}$ is analytic with a left-hand cut, and a right-hand cut which only starts at $t=t_{1}$. Therefore, $V_{0}^{0}$ is expected to be a smooth function in the region $\left[0, t_{1}\right]$ and we can use approximations by polynomials there. In practice, we used fourth order polynomials,

$$
V_{0}^{0}(t)=\alpha_{0}+\tilde{\beta}_{0} t+\tilde{\gamma}_{0} t^{2}+\delta_{0} t^{3}, \quad 0 \leq t \leq t_{f i t}<t_{1} .
$$


The first two parameters are determined from ChPT as above and we fit the remaining two to values of $V_{0}^{0}$ determined from the data above the $K \bar{K}$ threshold. The energy range in which the fit is performed $t \leq t_{f i t}$ cannot, of course, be made two large otherwise higher order polynomials would be needed. Fig. 4 shows two different fits and illustrates that this procedure, while simple, is also quite stable. This procedure allows one to determine

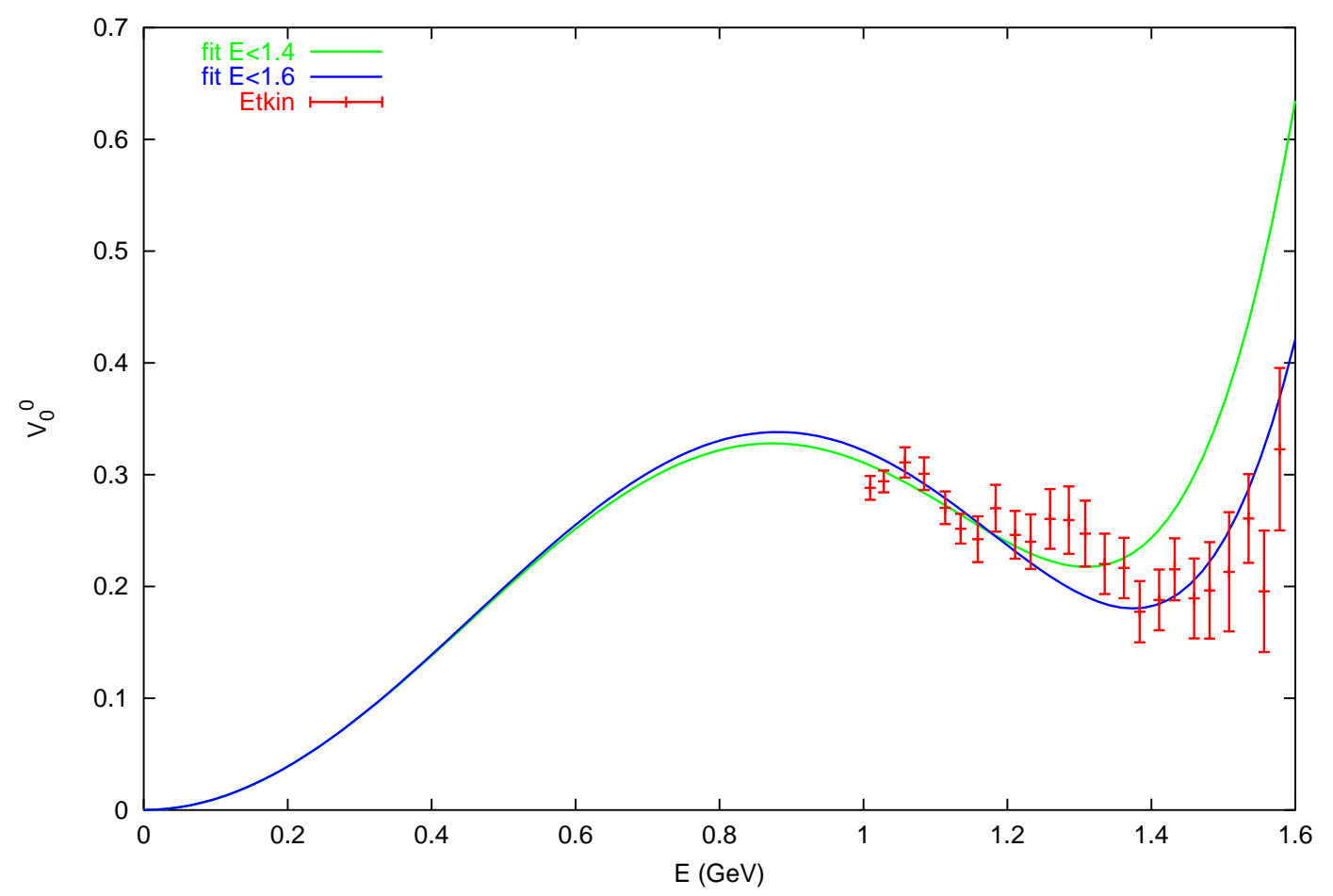

Figure 4: Polynomial fits of $g_{0}^{0}$ with the right-hand cut (partly) removed (see eq. (69)).

the value of $\left|g_{0}^{0}\left(4 m_{K}^{2}\right)\right|$ (which is thus correlated with the value of $\phi_{S}\left(4 m_{K}^{2}\right)$ ) and can be used for correctly computing the integrals above (64). We can also use the polynomial approximation to $V_{0}^{0}$ to extrapolate $g_{0}^{0}$ below the $K \bar{K}$ threshold (note that this method requires no knowledge of the left-hand cut and no assumption concerning asymptotic behaviour). We found that the two methods of extrapolation are in very good agreement.

The solution for $g_{0}^{0}$ has a rather strong dependence on the value of the phase $\phi_{S}$ at the $K \bar{K}$ threshold as is shown in fig. 5: the larger $\phi_{S}\left(4 m_{K}^{2}\right)$ the higher is the corresponding $f_{0}(980)$ resonance peak. Another source of uncertainty in this calculation is the fact that the two available data sets for $\left|g_{0}^{0}\right|$, while having small error bars, are not exactly compatible. The data of ref. [28] lies systematically below the data from ref. [29, 30]. The corresponding influence in the $f_{0}(980)$ peak is shown in fig. 6 .

Let us now turn to the $l=1$ amplitude $g_{1}^{1}(t)$. We will again here rely on the experimental data from ref. [28] above the $K \bar{K}$ threshold and chiral symmetry at $t=0$. Let 


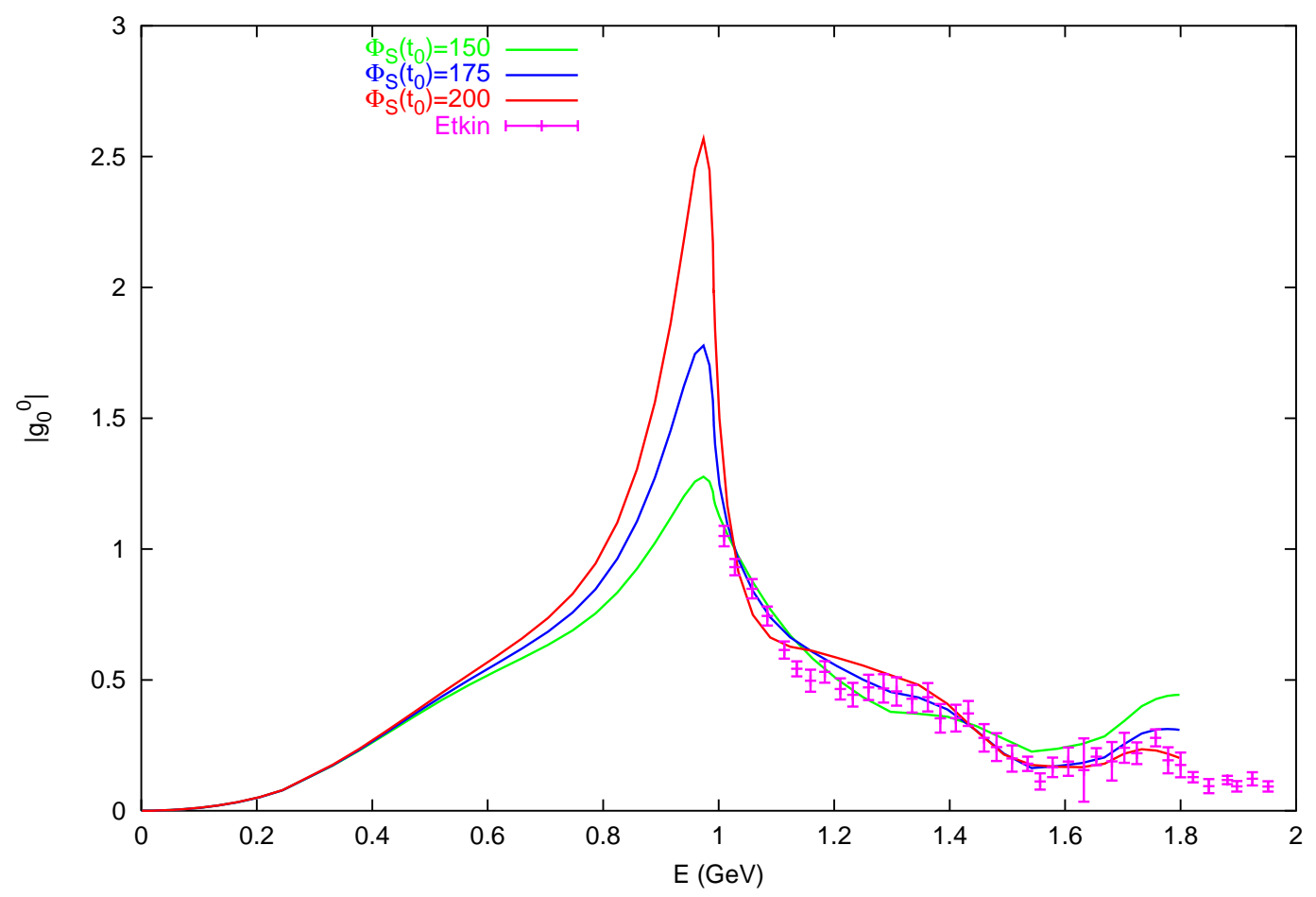

Figure 5: Comparison of several solutions of eq. (57) for $g_{0}^{0}(t)$ corresponding to different input values of the threshold phase $\phi_{S}\left(4 m_{K}^{2}\right)$.

us first consider the phase of $g_{1}^{1}$ which we denote by $\phi_{P}(t)$. In the range $\sqrt{t} \leq 0.82 \mathrm{GeV}$, $\phi_{P}(t)$ is equal to the $l=1 \pi \pi$ phase-shift and we use the parametrisation of ref. 32 which is constrained from the Roy equations. In the range $\sqrt{t} \geq 2 m_{K}$ the measured phase has been shown in ref. [28] to be well approximated by that of a Breit-Wigner tail of the following form

$$
g_{1}^{1}=\frac{m_{\rho} \sqrt{\hat{G}_{\pi}(t) \hat{G}_{K}(t)} / 2}{t-m_{\rho}^{2}-i m_{\rho}\left(G_{\pi}(t)+G_{K}(t)\right)}
$$

with

$$
\hat{G}_{\pi}(t)=\frac{m_{\rho} \Gamma_{\rho}}{q_{0}^{3}} \frac{1+R^{2} q_{0}^{2}}{1+R^{2} q_{\pi}^{2}}, \hat{G}_{K}(t)=\frac{m_{\rho} \Gamma_{\rho}}{2 q_{0}^{3}} \frac{1+R^{2} q_{0}^{2}}{1+R^{2} q_{K}^{2}}
$$

and

$$
G_{\pi}=\left(q_{\pi}^{3} / \sqrt{t}\right) \hat{G}_{\pi}, G_{K}=\left(q_{K}^{3} / \sqrt{t}\right) \hat{G}_{K}, q_{0}^{2}=m_{\rho}^{2} / 4-m_{\pi}^{2}, \quad R=3.5 \mathrm{GeV}^{-1} .
$$

The phase from this formula departs from the measured one above $1.6 \mathrm{GeV}$ but we will ignore this discrepancy as in this region the $l=1$ amplitude plays little role. Finally, in the intermediate region $0.82 \mathrm{GeV} \leq \sqrt{t} \leq 2 m_{K}$ we use the interpolation formula

$$
\tan \phi_{P}(t)=(a+b t) /\left(t-m_{\rho}^{2}\right), \quad 0.82 \mathrm{GeV} \leq \sqrt{t} \leq 2 m_{K}
$$




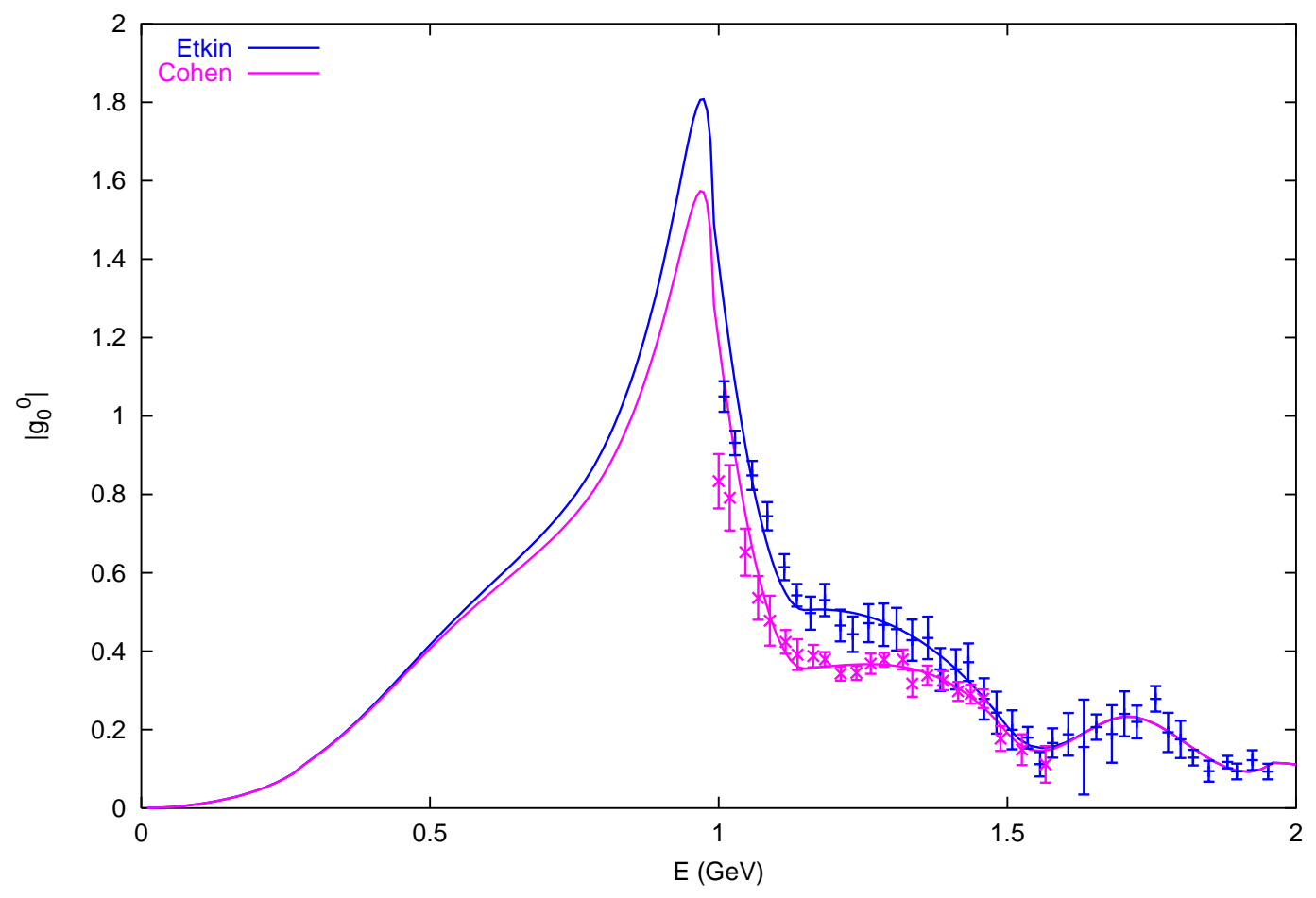

Figure 6: Comparison of two solutions for $\left|g_{0}^{0}\right|$ based on the two data sets of Cohen et al. 28] and Etkin et al. [29, 30] respectively. Above the $K \bar{K}$ threshold the curves are piecewise polynomial fits to the data and below the threshold they are obtained from eq. (64).

From this phase we can construct an Omnès function

$$
\Omega_{P}(t)=\exp \left[\frac{t}{\pi} \int_{4 m_{\pi}^{2}}^{\infty} \frac{\phi_{P}\left(t^{\prime}\right) d t^{\prime}}{t^{\prime}\left(t^{\prime}-t\right)}\right] .
$$

The magnitude of $g_{1}^{1}$ remains to be discussed. As in the case of $g_{0}^{0}$ we expect that it can be expressed with a good approximation as a low order polynomial times the Omnès function in the whole energy range of interest. In fact, earlier studies based on extrapolations away from the left-hand cut have shown that a constant polynomial is sufficient below one $\mathrm{GeV}$ [39, 40]. With this in mind, we made a fit to the data between 1 and $1.5 \mathrm{GeV}$ with a polynomial containing a constant term plus a term quadratic in $t$,

$$
g_{1}^{1}(t) \simeq \alpha_{1}\left(1+\beta_{1} t^{2}\right) \Omega_{P}(t), \sqrt{t} \leq 1.5 \mathrm{GeV}
$$

fixing $\alpha_{1}=\sqrt{2} / 48 \pi f_{\pi}^{2}$ from $O\left(p^{2}\right)$ chiral symmetry. A good fit is obtained in this way with $\beta_{1}=-0.187 \mathrm{GeV}^{-4}$ such that the quadratic term is indeed small below $1 \mathrm{GeV}$ (in discussing the errors we will introduce a linear term as well). The result for $\left|g_{1}^{1}\right|$ is shown in fig. 7. 


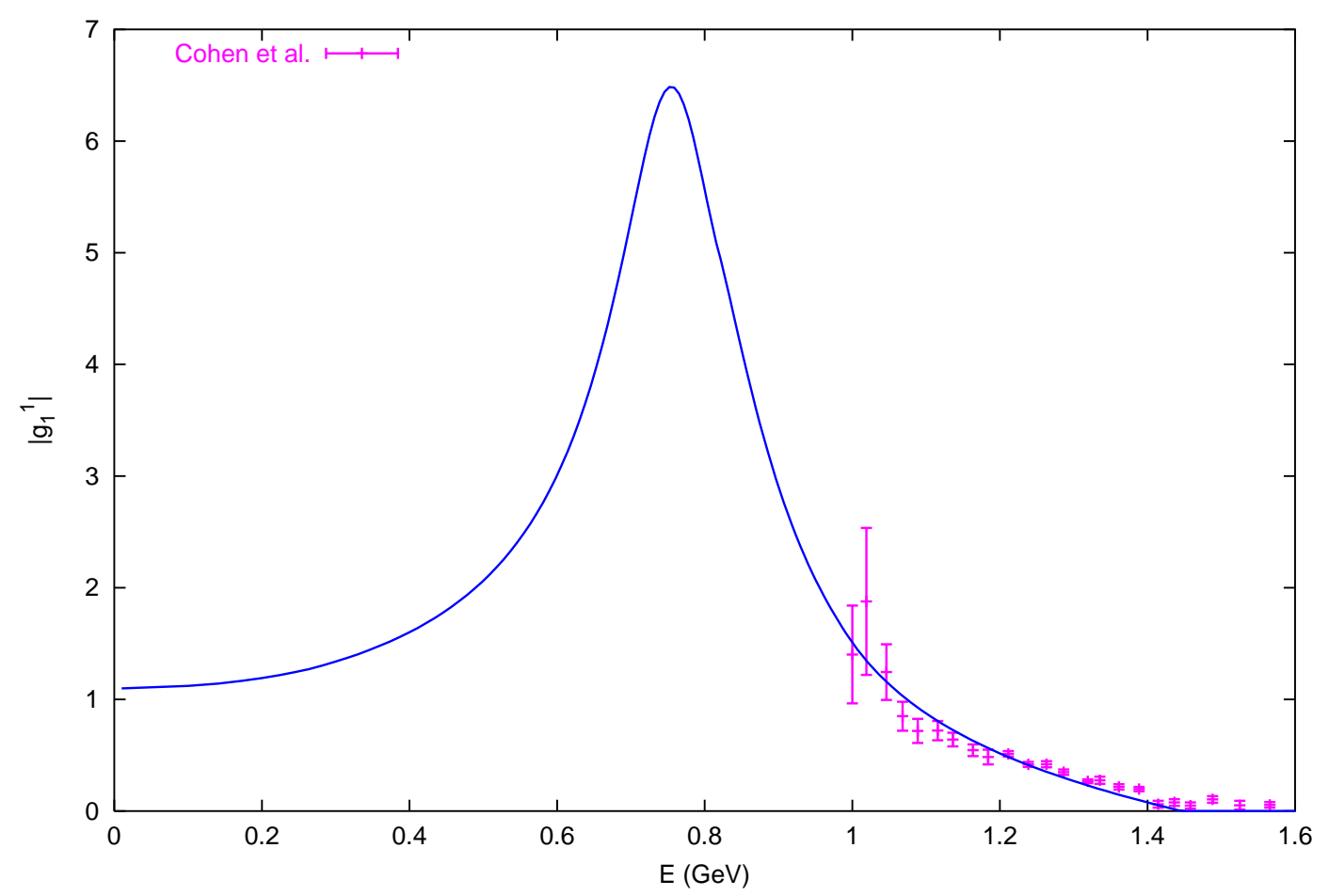

Figure 7: Magnitude of the $l=1$ partial wave $g_{1}^{1}$ (in units of $\mathrm{GeV}^{-2}$ ) from the construction described in the text, compared to the experimental data 28].

We have also included higher partial waves with $l=2,3$, 4. For $l=2$ we include the resonances $f_{2}(1270), f_{2}(1425), f_{2}(1810)$ with Breit-Wigner functions analogous to eq. (71) and parameters fitted to the data of ref. [30]. For $l=3$ we include $\rho_{3}(1690)$ and for $l=4$ the $f_{4}(2050)$ resonances. In both cases we take the $\pi \pi$ and $K \bar{K}$ partial widths from the PDG 41].

\subsection{Asymptotic region}

Beyond the energy region where the amplitudes are effectively measured in experiments we can hardly make better than qualitative estimates. For this purpose we will assume that the resonance region matches to a region where Regge behaviour prevails. More specifically, we will consider the dual-resonance model for the $\pi K$ amplitude (see e.g. 27 )

$$
\begin{aligned}
& F^{ \pm}(s, t, u)=-\lambda\left[V_{K^{*} \rho}(s, t) \pm V_{K^{*} \rho}(u, t)\right] \\
& V_{K^{*} \rho}(s, t)=\frac{\Gamma\left(1-\alpha_{K^{*}}(s)\right) \Gamma\left(1-\alpha_{\rho}(t)\right)}{\Gamma\left(1-\alpha_{K^{*}}(s)-\alpha_{\rho}(t)\right)} .
\end{aligned}
$$


For the Regge trajectories we take

$$
\alpha_{\rho}(t)=0.475+\alpha_{1} t, \quad \alpha_{K^{*}}(s)=0.352+\alpha_{1} s, \alpha_{1}=0.882 \mathrm{GeV}^{-2},
$$

and for the parameter $\lambda$ we take $\lambda=1.82$ which realises an approximate matching to the region known from experiment around $\sqrt{s}=2 \mathrm{GeV}$. In taking asymptotic limits in formula $(\sqrt{77})$ an $i \epsilon$ prescription is understood, for instance, $s \rightarrow \infty$ means $|s| \rightarrow \infty$ and $s=|s| \exp (i \epsilon)$. One then finds the well known Regge behaviour,

$$
\operatorname{Im} F^{ \pm}(s, t, u)_{s \rightarrow \infty, t \text { fixed }} \sim \frac{\pi \lambda}{\Gamma\left(\alpha_{\rho}(t)\right)}\left(\alpha_{1} s\right)^{\alpha_{\rho}(t)} .
$$

In the case of $F^{+}(s, t)$ one needs to include additionally the Pomeron,

$$
\operatorname{ImF}^{+}(s, 0)_{\text {Pomeron }}=\frac{1}{\pi} \sigma s,
$$

in which we take $\sigma=2.5 \mathrm{mb}$ (see the discussion in ref. [32]). We also need $\operatorname{ImF}^{ \pm}(s, t, u)$ in the regime where $t \rightarrow \infty$ and $s \rightarrow 0$ in the integrals $G_{b}^{ \pm}(n)$. The model (77) gives a Regge behaviour associated with the $K^{*}$ trajectory,

$$
\operatorname{Im} F^{ \pm}(s, t, u)_{t \rightarrow \infty, s \text { fixed }} \sim \frac{\pi \lambda}{\Gamma\left(\alpha_{K^{*}}(s)\right)}\left(\alpha_{1} t\right)^{\alpha_{K^{*}}(s)} .
$$

Finally, we need $\operatorname{ImF}^{ \pm}(s, t, u)$ in a regime where $s \rightarrow \infty$ and $u \rightarrow 0$ in the integrals $H_{b}^{ \pm}(n)$. In this case, the term $V_{K^{*} \rho}(u, t)$ in eq. (77) makes no contribution to the imaginary part (this, of course, reflects the exact degeneracy of the $K^{*}$ and $K_{2}^{*}$ trajectories in this model) and the term $V_{K^{*} \rho}(s, t)$ becomes exponentially suppressed (this term is the amplitude for the reaction $\pi^{+} K^{-} \rightarrow \pi^{+} K^{-}$and the corresponding $u$-channel is $\pi^{+} K^{+} \rightarrow \pi^{+} K^{+}$, which is exotic). The influence of these asymptotic contributions can be appreciated from table 1 below.

\begin{tabular}{|c|c|c|c|c|c|c|}
\hline & $H_{0}^{+}(2)$ & $H_{0}^{+}(3)$ & $H_{b}^{+}(2)$ & $H_{b}^{+}(3)$ & $G_{b}^{+}(2)$ & $G_{b}^{+}(3)$ \\
\hline \hline$\left[\Lambda^{2}, 4 \mathrm{GeV}^{2}\right]$ & 8.05 & 4.66 & 4.56 & 3.11 & 1.26 & 0.92 \\
\hline$\left[4 \mathrm{GeV}^{2}, \infty\right]$ & 8.75 & 0.72 & 0 & 0 & 1.17 & 0.13 \\
\hline \hline & $H_{0}^{-}(2)$ & $H_{0}^{-}(3)$ & $H_{b}^{-}(2)$ & $H_{b}^{-}(3)$ & $G_{b}^{-}(2)$ & $G_{b}^{-}(3)$ \\
\hline \hline$\left[\Lambda^{2}, 4 \mathrm{GeV}^{2}\right]$ & 5.81 & & 2.32 & 1.74 & 1.76 & 1.17 \\
\hline$\left[4 \mathrm{GeV}^{2}, \infty\right]$ & 2.18 & & 0 & 0 & 1.25 & 0.14 \\
\hline
\end{tabular}

Table 1: Results for the high-energy integrals (see eqs. (18) (28)) in appropriate powers of $\mathrm{GeV}$, showing two different integration regions.

\subsection{Results}

Let us first perform some simple checks. Equating two dispersive representations of $F^{-}(s, t)$ we obtained eq. (27): using this relation at $t=0$ gives one relation among the building blocks of the sum rules

$$
2 \hat{A}_{1}+H_{0}^{-}(2)=u_{1}+G_{b}^{-}(2)+H_{b}^{-}(2)
$$


We expect some uncertainty because of the relatively slow convergence in the integrals $H_{0}^{-}(2), G_{b}^{-}(2)$ but there is some amount of cancellation of these effects. Using the phenomenological input as described above, we obtain,

$$
2 \hat{A}_{1}+H_{0}^{-}(2) \simeq 33.17, \quad u_{1}+G_{b}^{-}(2)+H_{b}^{-}(2) \simeq 32.07\left[\mathrm{GeV}^{-2}\right] .
$$

Clearly, there is a very reasonable degree of agreement. This provides a check on the construction of the $\pi \pi \rightarrow K \bar{K} \mathrm{P}$-wave. We have also redone with our input the calculation of Karabarbounis and Shaw [42] which gives the difference in the scattering lengths $a_{0}^{1 / 2}-$ $a_{0}^{3 / 2}$

$$
m_{\pi}\left(a_{0}^{1 / 2}-a_{0}^{3 / 2}\right)=\frac{3 m_{\pi}}{8 \pi\left(m_{\pi}+m_{K}\right)} F^{-}\left(m_{+}^{2}, 0\right) \simeq 0.22
$$

to be compared with the result [42] $m_{\pi}\left(a_{0}^{1 / 2}-a_{0}^{3 / 2}\right)=0.26 \pm 0.05$. We have a comparable uncertainty due to a large extent to the asymptotic contributions. A related quantity is the polynomial parameter $\beta^{-}$(eq. (36)) for which a rather precise value is predicted by the chiral expansion,

$$
f_{\pi}^{2} \beta^{-}=0.25+0.01+O\left(p^{6}\right)
$$

where the successive contributions are shown. Using the sum rule expression (54) for $\beta^{-}$ we obtain

$$
f_{\pi}^{2} \beta^{-} \simeq 0.24
$$

which is within $10 \%$ of the result from ChPT. The size of the uncertainty in this calculation is approximately $15 \%$, so the agreement is satisfactory but it is not possible to separate the purely $O\left(p^{4}\right)$ part (in other term, we have no sum rule for $L_{5}$ ). We note also that the $O\left(p^{4}\right)$ contribution being suppressed, the $O\left(p^{6}\right)$ one could be of comparable size.

Let us now discuss the results for the chiral couplings $L_{1}, L_{2}, L_{3}$. We recall that these are obtained by first generating sum rules for the polynomial coefficients $\lambda_{1}^{+}, \lambda_{2}^{+}$, $\lambda_{1}^{-}$: here the contributions from the asymptotic regions are suppressed so we can expect rather good accuracy. Our results are collected in the last line of table 2, they complete and update those already given円 in ref. [15]. The way in which the errors quoted in the

\begin{tabular}{|c|c|c|c|c|}
\hline \multicolumn{1}{|c|}{$10^{3} \times$} & $L_{1}$ & $L_{2}$ & $L_{3}$ & $L_{3}+2 L_{1}$ \\
\hline \hline$\pi \pi$ sr $O\left(p^{4}\right)$ & - & $1.02 \pm 0.05$ & - & $-2.78 \pm 0.32$ \\
\hline$K_{l 4} r O\left(p^{4}\right)$ & $0.46 \pm 0.23$ & $1.49 \pm 0.23$ & $-3.18 \pm 0.85$ & $-2.26 \pm 0.97$ \\
\hline$\pi K$ sr $O\left(p^{4}\right)$ & $0.84 \pm 0.15$ & $1.36 \pm 0.13$ & $-3.65 \pm 0.45$ & $-1.97 \pm 0.34$ \\
\hline
\end{tabular}

Table 2: Results from the sum rules for the chiral couplings $L_{1}, L_{2}, L_{3}$ (multiplied by $10^{3}$ ) at the scale $\mu=0.770 \mathrm{GeV}$ (last line), compared to the results from $\pi \pi$ sum rules and from the $K_{l 4}$ form-factors.

table are evaluated will be explained in more details below, they do not include any effect

\footnotetext{
${ }^{1}$ In ref. [15] a factor $f_{\pi}^{2} f_{K}^{2}$ was used in eqs. (47) (48) instead of $f_{\pi}^{4}$. Here, we prefer not to include incomplete parts of the $O\left(p^{6}\right)$ contributions.
} 
from $O\left(p^{6}\right)$ corrections. The table also shows for comparison the results obtained from $\pi \pi$ sum rules (taken from ref. [34] in which $O\left(p^{4}\right)$ matching is used) and the results based on the $K_{l 4}$ form-factors also computed at chiral order $p^{4}$. The numbers quoted in the table are taken from the fit of Amoros et al. 2 [43] based on the data 3 of Rosselet et al. [44]. We note that the value of $L_{1}$ obtained previously in ref. [ 8$], 10^{3} L_{1}=0.65 \pm 0.27$, while compatible, has a somewhat larger central value. Our results for $L_{2}$ and $L_{3}$ agree within approximately $30 \%$ with the results from $K_{l 4}$ or $\pi \pi$. Concerning $L_{2}$, the difference beween the $K_{l 4}$ and the $\pi \pi$ result is substantially larger than that, our result happens to lie in between these two. A discrepancy at the $30 \%$ level can be expected as a consequence of unaccounted for $O\left(p^{6}\right)$ effects. In the case of $L_{1}$, however, there is a larger discrepancy, by about a factor of two, between our value and that from ref. 443]. The combination $L_{2}-2 L_{1}$ is suppressed in the large $N_{c}$ limit [四]. We indeed find a suppression of the value of $L_{2}-2 L_{1}$ (compared, say, with $L_{1}\left(m_{\rho}\right)$ or $L_{2}\left(m_{\rho}\right)$ ) even though this combination is dominated by scalar resonances. A calculation of the $K_{l 4}$ from factors in ChPT to order $p^{6}$ was recently performed 43, 45 and the couplings $L_{1}, L_{2}, L_{3}$ were then redetermined, using a model to estimate the $O\left(p^{6}\right)$ couplings $C_{i}(\mu)$. The following numbers are obtained 43.

$$
10^{3} L_{1}=0.53 \pm 0.25, \quad 10^{3} L_{2}=0.71 \pm 0.27, \quad 10^{3} L_{3}=-2.72 \pm 1.12 \quad\left[K_{l 4}, O\left(p^{6}\right)\right] .
$$

Clearly, variations larger than naively expected can occur as compared to the $O\left(p^{4}\right)$ determination. It remains to be seen, and this would be an interesting check of the convergence of the $S U(3)$ chiral expansion, how the differences in the results from the various methods of determining $L_{1}, L_{2}, L_{3}$ are reduced, once the $O\left(p^{6}\right)$ contributions are included.

In order to estimate the errors, firstly, we have varied the $S$ and $P$ wave $\pi K$ phaseshifts inside bands of half-width $\Delta \delta_{0}^{1 / 2}=2^{\circ}, \Delta \delta_{0}^{3 / 2}=1^{\circ}, \Delta \delta_{1}^{1 / 2}=1^{\circ}$, which correspond to the average experimental errors in the region of elastic scattering (which makes the most important contribution). For the $\pi \pi \rightarrow K \bar{K}$ P-wave, $g_{1}^{1}$, we have varied the coefficients of the normalising polynomial (see eq. (76)), allowing for a term linear in $t$, and requiring that the $\chi^{2}$ does not exceed twice its minimal value. The coefficient $\alpha_{1}$ is kept fixed since its variation can be considered as an $O\left(p^{6}\right)$ effect, which we do not try to estimate. This procedure generates a variation of the height of the $\rho$ resonance peak in the $10 \%$ range, which may seem rather small, but affects the results quite substantially, as can be seen from table 3 . For the $S$-wave, $g_{0}^{0}$, we have varied the phase at threshold $\phi_{S}\left(4 m_{K}^{2}\right)$ (which, as we have seen, is the parameter on which the size of the $f_{0}(980)$ peak mostly depends) in a range between 150 and 200 degrees. Above threshold we have made $\left|g_{0}^{0}\right|$ to vary in the whole range allowed by the two incompatible experiments. We have also allowed a $10 \%$ variation of the scattering lengths $a_{0}^{1 / 2}, a_{0}^{3 / 2}$ (keeping, however, the difference fixed) and, finally, in the Regge region we have assumed a $100 \%$ uncertainty. We show the individual impact of these variations on the sum rule results in table 3 .

We now come to the discussion of $L_{4}$. From relation (50) an important remark can be made concerning the convergence: while $\beta^{+}$and $\beta^{-}$, separately, contain integrals

\footnotetext{
${ }^{2}$ We thank P. Talavera for communicating the values of the errors corresponding to this fit.

${ }^{3}$ An indicative fit using preliminary data from the more recent E865 experiment [33] is performed in ref. 43] which gives essentially the same central values for $L_{1}, L_{2}, L_{3}$ and error bars reduced by approximately a factor of two.
} 


\begin{tabular}{|c|c|c|c|l|}
\hline $10^{5} \times$ & $\Delta L_{1}$ & $\Delta L_{2}$ & $\Delta L_{3}$ & $\Delta L_{4}$ \\
\hline \hline$\delta_{0}^{3 / 2}$ & 0.2 & 0.5 & 0.7 & 0.5 \\
\hline$\delta_{0}^{1 / 2}$ & 0.7 & 0. & 3.0 & 3.6 \\
\hline$\delta_{1}^{1 / 2}$ & 0.1 & 1.5 & 2.5 & 0.6 \\
\hline$a_{0}^{1 / 2}$ & 0.3 & 0.4 & 1.4 & 0.6 \\
\hline$g_{1}^{1}$ & 9. & 9. & 36. & 10. \\
\hline$g_{0}^{0}$ & 4.5 & 0. & 0. & 15. \\
\hline Regge & 0.3 & 1.6 & 1.0 & 0.1 \\
\hline
\end{tabular}

Table 3: List of different sources of errors (see text for details) and their impact on the determination of the $L_{i}$ 's.

which are slowly convergent at infinity, $L_{4}$ involves the difference, which has much better convergence properties. Indeed, consider the high-energy contribution

$$
\left[\beta^{+}-\beta^{-}\right]_{H E}=G_{b}^{+}(2)-G_{b}^{-}(2)-\left(H_{b}^{+}(2)+H_{b}^{-}(2)\right)+2 \Sigma_{\pi K}\left(H^{+}(3)-2 H_{b}^{+}(3)-H_{b}^{-}(3)\right) .
$$

On rather general grounds, the leading Regge contribution in $G_{b}^{+}(2)$ and $G_{b}^{-}(2)$ is the same and will cancel out in the difference. Also in the second potentially dangerous term $H_{b}^{+}(2)+H_{b}^{-}(2)$ the relevant cross channel is pure $I=3 / 2$ and has no leading Regge contributions. The other terms in eq. (88) are, as we have seen, rather insensitive to the asymptotic region. Therefore, we expect the uncertainty in $L_{4}$ coming from the asymptotic region to be small, of the same size as in $L_{1}, L_{2}, L_{3}$. Let us now consider the energy region below $1 \mathrm{GeV}$. Using eqs. (50) and (54) we can write,

$$
\left[L_{4}\right]_{L E}=2 A_{1}^{+}+2 \hat{A}_{1}^{+}-B_{0}^{+}-B_{0}^{-}+v_{0}-u_{1},+2 \Sigma_{\pi K}\left(-C_{0}^{+}-C_{0}^{-}+w_{0}\right)
$$

(where we have used $A_{1}^{-}=A_{1}^{+}, \hat{A}_{1}^{-}=\hat{A}_{1}^{+}$which is true up to $O\left(p^{8}\right)$ ). This expression contains $\mathrm{P}$-wave contributions, which may seem surprising in view of the well known resonance saturated expression [9]

$$
L_{4}^{r e s}=-\frac{c_{d} c_{m}}{3 M_{S_{8}}^{2}}+\frac{\tilde{c}_{d} \tilde{c}_{m}}{M_{S_{1}}^{2}}
$$

which involves only scalar resonances. It is in fact possible to write an alternative expression for (89): using the crossing symmetry relation (82) the P-wave combination $2 \hat{A}_{1}^{+}-u_{1}$ gets replaced by contributions from above the resonance region and the $\mathrm{P}$-wave term $A_{1}^{+}$ has, in fact, no contribution from the resonances. This alternative expression has only $\mathrm{S}$-wave resonance contributions but is not as rapidly convergent.

Numerical results for $L_{4}$ are shown in table 4 for several input values of the threshold phase $\phi_{S}\left(4 m_{K}^{2}\right)$. We have also mentioned that the two experimental measurements of $\left|g_{0}^{0}\right|$ of refs. [28] and [30] have a somewhat inconsistent normalisation. We have performed the calculation for each data set separately. A clear feature from these calculations is that $L_{4}\left(m_{\rho}\right)$ is suppressed, and has a magnitude similar to $2 L_{1}-L_{2}$. It is not very 


\begin{tabular}{|c|c|c|c|c|c|l|}
\hline$\phi_{S}\left(4 m_{K}^{2}\right)$ & $150^{\circ}$ & $175^{\circ}$ & $185^{\circ}$ & $200^{\circ}$ & $220^{\circ}$ & \\
\hline $10^{3} L_{4}$ & 0.08 & 0.18 & 0.22 & 0.27 & 0.34 & Etkin \\
& 0.03 & 0.10 & 0.13 & 0.16 & 0.21 & Cohen \\
\hline
\end{tabular}

Table 4: Sum rule results for $L_{4}(\mu=0.770)$.

easy to decide on which central value to choose. We will make the choice of believing the data of Cohen et al. [28] for the value of $\phi_{S}\left(4 m_{K}^{2}\right)$, which is then close to $200^{\circ}$, as they argue that the presence of a $\mathrm{P}$-wave in their experiment (which is absent in the other experiment) helps in correctly determining the S-wave phase at threshold. Concerning the normalisation of $\left|g_{0}^{0}\right|$ above the threshold we may average over both experiments. Taking into account the main sources of uncertainty (see table 3) we would then obtain,

$$
L_{4}(\mu=0.770)=(0.22 \pm 0.30) \cdot 10^{-3} .
$$

This result is a refinement of the previous estimate of Gasser and Leutwyler [4], $L_{4}(\mu=$ $0.770)=(-0.3 \pm 0.5) \cdot 10^{-3}$, based on the assumption that OZI suppression holds but without precisely knowing the value of the scale at which it does. Other results can be found in the literature [46, 47] which, however, are based on some assumptions allowing one to determine scalar form-factors. The fact that they agree with (91) indicates that these assumptions are reasonable. Finally, Amoros et al. [43] have attempted to determine $L_{4}$ from $K_{l 4}$ data, using their $O\left(p^{6}\right)$ calculations, and they obtain $L_{4}(\mu=0.770)=$ $(-0.2 \pm 0.9) \cdot 10^{-3}$ which, as expected, is not very tightly constrained.

\section{Conclusions}

In this paper we have performed an evaluation of the set of sum rules proposed in ref. [15]. While some results were already presented in ref. [15], the calculations performed here are more complete: they take into account the contributions from partial waves beyond the $\mathrm{S}$ and $\mathrm{P}$ waves as well as asymptotic contributions. In order to fully exploit these sum rules, one needs to perform an extrapolation of the $l=0$ and $l=1$ partial waves of the $\pi \pi \rightarrow K \bar{K}$ amplitude. This can be performed using standard MuskhelishviliOmnès techniques and was considered a long time ago [16]. A great improvement over these calculations is the availability nowadays of direct and precise experimental results concerning the $\pi \pi \rightarrow K \bar{K}$ amplitude, so there is no need to make use of inelasticity in $\pi \pi$ scattering, which is not very precisely determined, and requires the assumption of exact two-channel unitarity. We have checked the stability of the calculation by comparing different approaches to the solution. The main source of uncertainty comes from the value of the phase at the $K \bar{K}$ threshold because the height of the $f_{0}(980)$ peak is strongly correlated with the value of this phase. The two available experimental data sets do not agree on this value and we have assumed a plausible range of variation. We have obtained a redetermination of the three $O\left(p^{4}\right)$ chiral couplings $L_{1}, L_{2}, L_{3}$. Comparison with former determinations allows a test of the $S U(3)$ chiral expansion. For instance, it is encouraging that the value of $L_{2}$ that we obtain is intermediate between the value from $K_{l 4}$ and the value from $\pi \pi$ sum rules. Besides, we have obtained for the first time an evaluation of $L_{4}$ 
at the same level of precision and reliability as $L_{1}, L_{2}, L_{3}$. This was not possible from the $K_{l 4}$ from-factors because of an accidental suppression of the coefficient of $L_{4}$ in this case.

In constructing the $l=0$ and $l=1$ partial waves of the $\pi \pi \rightarrow K \bar{K}$ amplitude, we have solved a subset of the system of Roy-Steiner equations. A further improvement, which we have not performed here, would be to use the full set of equations in order to constrain the low energy part of the $\pi K \rightarrow \pi K$ amplitudes. We note however, that the range of energies where this is needed, that is, between the threshold and the energy where the data start is smaller for $\pi K$ than it is for $\pi \pi$. Solving these equations would help in deciding whether a strange counterpart of the $\sigma$ meson, the $\kappa$ meson, actually exists (e.g. ref. [11] and references therein) or not [48]. An obvious further improvement would be to use the sum rules in association with a chiral $O\left(p^{6}\right)$ calculation of the $\pi K$ amplitudes. These, taken together with the $\pi \pi$ sum rules (associated also with an $O\left(p^{6}\right) S U(3)$ calculation of the $\pi \pi$ amplitude) and the available calculation of the $K_{l 4}$ form-factors to this order 45 would no doubt greatly improve our understanding of the chiral expansion in $m_{s}$.

Acknowledgements B.M. would like to thank B. Nicolescu for tuition on Regge physics, M. Pennington for correspondence, W. Dunwoodie and R.S. Longacre for providing informations on the data. P.B. would like to thank IPN Orsay for its hospitality and financial support during his stay in Paris.

This work is supported in part by the EEC-TMR contract ERBFMRXCT98-0169, by IFCPAR contract 2504-1 and DFG under contract np. ME 864-15/2. 


\section{References}

[1] J. Bijnens, G. Colangelo and G. Ecker, Annals Phys. 280 (2000) 100 hepph/9907333; JHEP 9902 (1999) 020 hep-ph/9902437.

[2] S. Weinberg, PhysicaA 96 (1979) 327.

[3] J. Gasser and H. Leutwyler, Annals Phys. 158 (1984) 142.

[4] J. Gasser and H. Leutwyler, Nucl. Phys. B 250 (1985) 465.

[5] G. Ecker, Prog. Part. Nucl. Phys. 35 (1995) 1 [hep-ph/9501357].

[6] V. Bernard, N. Kaiser and U. G. Meißner, Phys. Rev. D 43 (1991) 2757; Nucl. Phys. B 357 (1991) 129.

[7] J. Bijnens, Nucl. Phys. B 337 (1990) 635;

[8] C. Riggenbach, J. Gasser, J. F. Donoghue and B. R. Holstein, Phys. Rev. D 43 (1991) 127.

[9] G. Ecker, J. Gasser, A. Pich and E. de Rafael, Nucl. Phys. B 321 (1989) 311.

[10] Y. Iwasaki, K. Kanaya, S. Kaya, S. Sakai and T. Yoshie, Prog. Theor. Phys. Suppl. 131 (1998) 415 hep-lat/9804005]; R. D. Mawhinney, Nucl. Phys. Proc. Suppl. 60A (1998) 306 hep-lat/9705031.

[11] D. Black, A. H. Fariborz, F. Sannino and J. Schechter, Phys. Rev. D 58 (1998) 054012 hep-ph/9804273.

[12] A. Roessl, Nucl. Phys. B 555 (1999) 507 hep-ph/9904230.

[13] M. Jamin, J. A. Oller and A. Pich, Nucl. Phys. B 587 (2000) 331 hep-ph/0006045.

[14] P. Minkowski and W. Ochs, Eur. Phys. J. C 9 (1999) 283 hep-ph/9811518.

[15] B. Ananthanarayan and P. Büttiker, Eur. Phys. J. C 19 (2001) 517 hep-ph/0012023.

[16] N. O. Johannesson and J. L. Petersen, Nucl. Phys. B 68 (1974) 397.

[17] N. Johannesson and G. Nilsson, Nuovo Cim. A 43 (1978) 376.

[18] C. B. Lang, Fortsch. Phys. 26 (1978) 509.

[19] J. Stern, H. Sazdjian and N. H. Fuchs, Phys. Rev. D 47 (1993) 3814 hep-ph/9301244.

[20] W. R.Frazer andJ. R. Fulco, Phys. Rev. 117 (1960) 1603

[21] G. E. Hite and F. Steiner, Nuovo Cim. A 18 (1973) 237.

[22] M. Knecht, B. Moussallam, J. Stern and N. H. Fuchs, Nucl. Phys. B 471 (1996) 445 hep-ph/9512404. 
[23] P. Estabrooks, R. K. Carnegie, A. D. Martin, W. M. Dunwoodie, T. A. Lasinski and D. W. Leith, Nucl. Phys. B 133 (1978) 490.

[24] D. Aston et al., Nucl. Phys. B 296 (1988) 493.

[25] N. Hedegaard-Jensen, Nucl. Phys. B 77 (1974) 173.

[26] J. P. Ader, C. Meyers and B. Bonnier, Phys. Lett. B 46 (1973) 403.

[27] B. R. Martin, D. Morgan and G. Shaw, "Pion-Pion Interactions In Particle Physics," Academic Press, London, 1976.

[28] D. Cohen, D. S. Ayres, R. Diebold, S. L. Kramer, A. J. Pawlicki and A. B. Wicklund, Phys. Rev. D 22 (1980) 2595.

[29] A. Etkin et al., Phys. Rev. D 25 (1982) 1786.

[30] S. J. Lindenbaum and R. S. Longacre, Phys. Lett. B 274 (1992) 492.

[31] K. L. Au, D. Morgan and M. R. Pennington, Phys. Rev. D 35 (1987) 1633.

[32] B. Ananthanarayan, G. Colangelo, J. Gasser and H. Leutwyler, hep-ph/0005297.

[33] P. Truol et al. [BNL-E865 Collaboration], hep-ex/0012012, S. Pislak et al. [BNL-E865 Collaboration], hep-ex/0106071.

[34] G. Colangelo, J. Gasser and H. Leutwyler, hep-ph/0103088.

[35] N. Muskhelishvili, "Singular Integral Equations," P.Noordhof, Groningen, 1953.

[36] R. Omnes, Nuovo Cim. 8 (1958) 316.

[37] S. M. Roy, Phys. Lett. B 36 (1971) 353.

[38] F. Steiner, Fortsch. Phys. 18 (1970) 43; Fortsch. Phys. 19 (1971) 115.

[39] H. Nielsen and G. C. Oades, Nucl. Phys. B 55 (1973) 301.

[40] S. Blatnik, J. Stahov and C. B. Lang, Nuovo Cim. A 48 (1978) 107.

[41] D. E. Groom et al. [Particle Data Group Collaboration], Eur. Phys. J. C 15 (2000) 1.

[42] A. Karabarbounis and G. Shaw, J. Phys. G G6 (1980) 583.

[43] G. Amoros, J. Bijnens and P. Talavera, Nucl. Phys. B 602 (2001) 87 hep$\mathrm{ph} / 0101127$.

[44] L. Rosselet et al., Phys. Rev. D 15 (1977) 574.

[45] G. Amoros, J. Bijnens and P. Talavera, Phys. Lett. B 480 (2000) 71 hep-ph/9912398, G. Amoros, J. Bijnens and P. Talavera, Nucl. Phys. B 585 (2000) 293 [Erratum-ibid. B 598 (2000) 665] hep-ph/0003258. 
[46] B. Moussallam, Eur. Phys. J. C 14 (2000) 111 hep-ph/9909292, B. Moussallam, JHEP 0008 (2000) 005 hep-ph/0005245.

[47] U. G. Meißner and J. A. Oller, Nucl. Phys. A 679 (2001) 671 hep-ph/0005253.

[48] S. N. Cherry and M. R. Pennington, Nucl. Phys. A 688 (2001) 823 hep-ph/0005208. 